TITLE:

\title{
Asymptotic far-field behavior of macroscopic quantities in a problem of slow uniform rarefied gas flow past a sphere
}

\author{
$\operatorname{AUTHOR(S):~}$ \\ Taguchi, Satoshi; Suzuki, Toshihiro
}

\section{CITATION:}

Taguchi, Satoshi ... [et al]. Asymptotic far-field behavior of macroscopic quantities in a problem of slow uniform rarefied gas flow past a sphere. Physical Review Fluids 2017, 2(11): 113401.

ISSUE DATE:

2017-11

URL:

http://hdl.handle.net/2433/252788

RIGHT:

(C) 2017 American Physical Society 


\title{
Asymptotic far-field behavior of macroscopic quantities in a problem of slow uniform rarefied gas flow past a sphere
}

\author{
Satoshi Taguchi* \\ Department of Advanced Mathematical Sciences, Graduate School of Informatics, \\ Kyoto University, Kyoto 606-8501, Japan \\ Toshihiro Suzuki ${ }^{\dagger}$ \\ Department of Mechanical and Intelligent Systems Engineering, \\ The University of Electro-Communications, Chofu, Tokyo 182-8585, Japan
}

(Received 17 May 2017; published 17 November 2017)

\begin{abstract}
The steady behavior of a low Mach number flow of a rarefied gas past a sphere is considered on the basis of the linearized Boltzmann equation with a special interest in the asymptotic behaviors of the flow velocity and temperature in the region far from the sphere. The study is motivated by the previous one [Taguchi, J. Fluid Mech. 774, 363 (2015)], in which an asymptotic analysis of the Boltzmann equation for small Mach numbers was carried out to derive the expression of the drag up to the second order of the Mach number. The derived expression contains two functions of the Knudsen number, corresponding to the leading-order drag obtained from the linearized problem and the second-order correction due to the weak nonlinear effect. This correction is also obtained through the analysis of the linearized problem; it is given by the factor of the term in the flow velocity whose magnitude is inversely proportional to the distance from the sphere and therefore vanishes at infinity. In this study, this factor (and thus the correction) is obtained for a wide range of the Knudsen number for the hard-sphere gas as well as for the ellipsoidal statistical (ES) model of the Boltzmann equation, under the conventional diffuse reflection boundary condition. The construction is based on the universal relation between the linear drag and the factor (correction). With the available data for the linear drag, this allows us to derive the latter from the former. For the ES model with the Prandtl number $\operatorname{Pr}=2 / 3$, a series of additional numerical computations of the linearized problem is carried out to obtain the linear drag and then the correction. It is also shown that a factor occurring in the temperature, decaying in proportion to the inverse square of the distance from the sphere, is connected to the thermal force exerted on a sphere (thermophoresis), whose numerical values on the basis of the ES model are obtained as well.
\end{abstract}

DOI: 10.1103/PhysRevFluids.2.113401

\section{INTRODUCTION}

In this paper, we are concerned with a slow motion of a rarefied gas past a sphere held at rest in the gas, and in particular, the drag exerted on the sphere. The question is clearly among the most fundamental ones in fluid mechanics, and the asymptotic formula of the drag for small Reynolds numbers is well known for the continuum flow (or the Navier-Stokes flow) (e.g., Refs. [1-3]). For rarefied gas flows, however, the analysis is more complicated and the level of our understanding is still unsatisfactory. As a matter of fact, most of the analysis is limited to the linearized case, based

\footnotetext{
*taguchi.satoshi.5a@kyoto-u.ac.jp; also at Advanced Research Institute of Fluid Science and Engineering, Kyoto University.

${ }^{\dagger}$ Present address: Kyokuto Kaihatsu Kogyo Co., Ltd., 4-1-62 Fukaminishi, Yamato, Kanagawa 242-0018, Japan.
} 


\section{SATOSHI TAGUCHI AND TOSHIHIRO SUZUKI}

on the linearized equation and boundary conditions under the assumption of the small Mach number (e.g., Refs. [4-12]), and the result based on the nonlinear equation is quite few [13].

In the previous paper [14], the first author carried out an asymptotic analysis for small Mach numbers on the basis of the Boltzmann equation and its kinetic boundary condition, and derived an expression of the drag up to the second order of the (small) Mach number. The derived formula contains two functions, later denoted by $h_{D}$ and $c_{1}$, which essentially depend on the Knudsen number. Here, the Knudsen number is defined as the ratio between the molecular mean free path and the size of the sphere, and represents the degree of gas rarefaction. The first one, $h_{D}$, corresponds to the drag exerted on the sphere in the linearized problem (under the condition of the smallness of the Mach number), and thus the existing results of the linearized analysis are available for certain molecular models. The second one, $c_{1}$, which appears in the drag formula at the second order level, gives the correction to the linear drag $\left(h_{D}\right)$ due to the weak nonlinear effect. Strikingly, this higher order correction is also derived from the analysis of the same problem as $h_{D}$, i.e., the leading-order linearized problem; $c_{1}$ is given by the factor of the term in the flow velocity whose magnitude is inversely proportional to the distance from the sphere and therefore vanishes at infinity. Up to now, the information on $c_{1}$ is limited to some preliminary results reported in Ref. [14] for the case of the Bhatnagar-Gross-Krook (BGK) model $[15,16]$ of the Boltzmann equation under the diffuse reflection boundary condition. In this paper, we construct the function $c_{1}$ in the case of the hard-sphere gas as well as in the case of the ellipsoidal statistical (ES) model [17,18] under the diffuse reflection boundary condition, both falling into the class of the most utilized models in kinetic theory.

The key to construct $c_{1}$ is the universal relation between $h_{D}$ (i.e., the linear drag) and $c_{1}$ (i.e., the factor or correction). Owing to this relation, one can readily obtain the latter once we know the former (the functional dependency of $h_{D}$ on the Knudsen number). This applies to the case of the hard-sphere gas, where the accurate numerical values of $h_{D}$ are available in Ref. [11]. For the ES model, we were not able to find any reported value of $h_{D}$, except for the case of the Prandtl number $\operatorname{Pr}=1$, for which the model reduces to the BGK model. Therefore, for the ES model, we first solve the linearized problem to obtain $h_{D}$ as a function of the Knudsen number, and then $c_{1}$ with the aid of the mentioned relation.

In this paper, we also investigate the behavior of the temperature far from the sphere in the linearized problem. More precisely, we are interested in the factor, later denoted by $c_{3}$, of the temperature, whose magnitude decays in proportion to the inverse square of the distance from the sphere. We see that $c_{3}$ is directly connected to the force exerted on a sphere in the problem of thermophoresis, say, $h_{T}$, which is seen to be the counterpart of the above-mentioned relation between $h_{D}$ and $c_{1}$.

The paper is organized as follows. In Sec. II we summarize the result of the asymptotic analysis carried out in Ref. [14]. In particular, we introduce two functions $h_{D}$ and $c_{1}$ and express the drag in terms of these functions. Also introduced is a factor $c_{3}$ of the term describing the temperature far from the sphere in the linearized problem. Section III is devoted to the actual construction of $c_{1}$ and $c_{3}$ as functions of the Knudsen number. We show that there are simple relations between $h_{D}$ and $c_{1}$ and between $h_{T}$ and $c_{3}$. With the aid of these relations and available data for $h_{D}$ and $h_{T}$, we obtain $c_{1}$ and $c_{3}$ for the hard-sphere gas. Subsequently, we construct $c_{1}$ and $c_{3}$ for the ES model. We first carry out a numerical analysis of the linearized problem to obtain the corresponding values of $h_{D}$, from which $c_{1}$ is immediately obtained. As for $c_{3}$, it is obtained with the aid of a representation formula, expressing $c_{3}$ in terms of the quantities on the boundary in the linearized problem. Section IV is the conclusion.

\section{SUMMARY OF ASYMPTOTIC ANALYSIS FOR SMALL MACH NUMBERS}

In this section, we summarize the main result of the asymptotic analysis for small Mach numbers for readers' convenience as well as for the sake of clarity of subsequent discussions. Let us begin with the statement of the problem considered in Ref. [14]. 


\section{A. Nonlinear problem}

Let us consider a flow of monatomic ideal gas past a sphere with radius $L$, held at rest in the gas. At infinity, the state of the gas is uniform with velocity $\left(v_{\infty}, 0,0\right)$, density $\rho_{\infty}$, and temperature $T_{\infty}$ (or pressure $p_{\infty}=R \rho_{\infty} T_{\infty}$, where $R=k_{B} / m$ is the specific gas constant with $k_{B}$ and $m$ being the Boltzmann constant and the mass of a molecule, respectively). The sphere is kept at the same temperature as the uniform flow. Investigate the steady behavior of the gas around the sphere, with a special interest in the drag exerted on the sphere by the flow, under the following assumptions:

(i) The behavior of the gas is described by the Boltzmann equation for a hard-sphere gas or by the ellipsoidal statistical model of the Boltzmann equation. (The general discussion in the sequel, however, is made for the general collision operator, not restricted to these models.)

(ii) The gas molecules are reflected according to the diffuse reflection condition on the surface of the sphere. That is, the velocity distribution of the molecules leaving the surface constitute the corresponding part of the Maxwellian characterized by the surface temperature, surface velocity, and the condition that there is no net mass flux across the boundary.

(iii) The Mach number Ma of the flow is small: $\mathrm{Ma}=v_{\infty} /\left(5 R T_{\infty} / 3\right)^{1 / 2} \ll 1$.

(iv) The Knudsen number $\mathrm{Kn}$ is finite: $\mathrm{Kn}=\ell_{\infty} / L=O(1)$.

Here, $\ell_{\infty}$ is the mean free path of the gas molecules in the equilibrium state at rest with temperature $T_{\infty}$ and pressure $p_{\infty}$. For a hard-sphere gas, $\ell_{\infty}$ is given by $\ell_{\infty}=1 / \sqrt{2} \pi d^{2}\left(\rho_{\infty} / m\right)$ with $d$ being the diameter of a molecule. For the ES model, $\ell_{\infty}=(2 / \sqrt{\pi})\left(2 R T_{\infty}\right)^{1 / 2} / A_{c} \rho_{\infty}$ with $A_{c}$ being a positive constant ( $A_{c} \rho_{\infty}$ is the collision frequency). In this paper, we mainly use the parameters $\epsilon$ and $k$, corresponding to the Mach number Ma and the Knudsen number $\mathrm{Kn}$, respectively:

$$
\begin{aligned}
& \epsilon=\frac{v_{\infty}}{\left(2 R T_{\infty}\right)^{1 / 2}}=\left(\frac{5}{6}\right)^{1 / 2} \mathrm{Ma}, \\
& k=\frac{\sqrt{\pi}}{2} \mathrm{Kn} .
\end{aligned}
$$

Corresponding to the assumptions (iii) and (iv), we have

$$
\epsilon \ll 1, \quad k=O(1)
$$

Some basic notations are introduced here. $L x_{i}$ is the space rectangular coordinates, $\left(2 R T_{\infty}\right)^{1 / 2} \zeta_{i}$ is the molecular velocity, $\rho_{\infty}\left(2 R T_{\infty}\right)^{-3 / 2}\left[1+\phi\left(x_{i}, \zeta_{i}\right)\right] E$ is the velocity distribution function (VDF) of the gas molecules. Here, $E=\pi^{-3 / 2} \exp \left(-\zeta_{j}^{2}\right)$. Further, $\rho_{\infty}(1+\omega)$ denotes the density, $\left(2 R T_{\infty}\right)^{1 / 2} u_{i}$ the flow velocity, $T_{\infty}(1+\tau)$ the temperature, $p_{\infty}(1+P)$ the pressure, $p_{\infty}\left(\delta_{i j}+P_{i j}\right)$ the stress tensor, and $p_{\infty}\left(2 R T_{\infty}\right)^{1 / 2} Q_{i}$ the heat-flow vector of the gas. We also use the spherical coordinate system $(r, \theta, \varphi)$ centered at the origin and with the polar axis oriented to the positive $x_{1}$ direction, i.e., $x_{1}=r \cos \theta, x_{2}=r \sin \theta \cos \varphi$, and $x_{3}=r \sin \theta \sin \varphi$. The components of vectors or tensors in the spherical coordinate system are indicated by the subscripts $(r, \theta, \varphi)$.

Then, our problem is to find the solution $\phi$ of the following boundary-value problem:

$$
\begin{gathered}
\zeta_{i} \frac{\partial \phi}{\partial x_{i}}=\frac{1}{k}(\mathcal{L}[\phi]+\mathcal{J}[\phi, \phi]), \\
\phi\left(r=1, \zeta_{r}>0\right)=-2 \sqrt{\pi} \int_{\zeta_{r}<0} \zeta_{r} \phi E d \zeta, \\
\phi \rightarrow \pi^{-3 / 2} \exp \left(-\left(\zeta_{i}-\epsilon \delta_{i 1}\right)^{2}\right) E^{-1}-1, \quad(r \rightarrow \infty),
\end{gathered}
$$

where $\mathcal{L}$ and $\mathcal{J}$ are the linear and nonlinear parts of the collision integral, respectively, $d \zeta=$ $d \zeta_{1} d \zeta_{2} d \zeta_{3}$, and $\delta_{i j}$ is the Kronecker delta. We give further information on $\mathcal{L}$ and $\mathcal{J}$ in Appendix A. 


\section{SATOSHI TAGUCHI AND TOSHIHIRO SUZUKI}

The macroscopic quantities are defined as

$$
\begin{aligned}
\omega & =\langle\phi\rangle, \quad(1+\omega) u_{i}=\left\langle\zeta_{i} \phi\right\rangle, \quad \frac{3}{2}(1+\omega) \tau=\left\langle\left(\zeta_{j}^{2}-\frac{3}{2}\right) \phi\right\rangle-(1+\omega) u_{j}^{2}, \\
P & =\omega+\tau+\omega \tau, \quad P_{i j}=2\left\langle\zeta_{i} \zeta_{j} \phi\right\rangle-2(1+\omega) u_{i} u_{j}, \\
Q_{i} & =\left\langle\zeta_{i} \zeta_{j}^{2} \phi\right\rangle-\frac{5}{2} u_{i}-u_{j} P_{i j}-\frac{3}{2} P u_{i}-(1+\omega) u_{i} u_{j}^{2},
\end{aligned}
$$

where

$$
\left\langle g\left(\zeta_{i}\right)\right\rangle=\int g E d \zeta
$$

and the integral with respect to $\zeta_{i}$ is carried out over the whole space.

In this paper, we exclusively consider the case of the diffuse reflection boundary condition [i.e., Eq. (4)]. Use of other boundary conditions is also possible and will affect the numerical values of $h_{D}, c_{1}, c_{2}$, and $c_{3}$ appearing below.

\section{B. Asymptotic analysis}

In Ref. [14], the problem was investigated by the method of matched asymptotic expansion for small $\epsilon$. In this section, we summarize the result of the analysis. In particular, we list the problems to determine the first two terms of the inner solution, describing the behavior of the gas near the sphere.

\section{Inner and outer solutions}

The primary length scale of the present problem is the size of the sphere, i.e., $L$. On the other hand, it was shown in Ref. [14] that there appears another length scale, characterized by $L / \epsilon$, in the region far from the sphere. We thus consider two regions, near and far regions, and introduce two types of solutions with different length scales, called the inner and outer (or slowly varying) solutions and denoted by $\phi_{I}$ and $\phi_{O}$, respectively. The length scales of variation of $\phi_{I}$ and $\phi_{O}$ are, respectively, 1 and $1 / \epsilon$ in the (dimensionless) $x_{i}$ space, i.e., $\partial \phi_{I} / \partial x_{i}=O\left(\phi_{I}\right)$ and $\partial \phi_{O} / \partial x_{i}=O\left(\epsilon \phi_{O}\right)$. We also suppose that the ranges of validity of $\phi_{I}$ and $\phi_{O}$ are $(1<) r \ll 1 / \epsilon$ and $r \gg 1$, respectively. This assumption is consistent with the following analysis. Thus, they overlap in the region characterized by $1 \ll r \ll 1 / \epsilon$, where the two solutions should coincide each other.

In Ref. [14], $\phi_{I}$ and $\phi_{O}$ are looked for in the form

$$
\phi_{J}=\phi_{J(1)} \epsilon+\phi_{J(2)} \epsilon^{2}+o\left(\epsilon^{2}\right), \quad(J=I, O),
$$

where $o\left(\epsilon^{2}\right)$ represents terms smaller than the second order of $\epsilon$. Note that the above expansion starts from $\epsilon$ order, since we are concerned with the solution whose order of magnitude is of the order of $\epsilon$ [assumption (iii)]. Likewise, the corresponding macroscopic quantities $h_{I}$ and $h_{O}\left(h=\omega, u_{i}, \tau\right.$, etc.) are also expanded as

$$
h_{J}=h_{J(1)} \epsilon+h_{J(2)} \epsilon^{2}+o\left(\epsilon^{2}\right), \quad(J=I, O) .
$$

Our main interest here is to determine the first two terms of the inner expansions $\phi_{I(1)}$ and $\phi_{I(2)}$, from which we compute the drag exerted on the sphere.

\section{Summary of the outer solution}

Before we present the problems to determine the inner solution, we first summarize the result for the outer (slowly varying) solution. According to Ref. [14], $\phi_{O(m)}$ and $h_{O(m)}$ are given, up to $m=2$, as follows:

Order $\epsilon$ :

$$
\begin{gathered}
P_{O(1)}=\tau_{O(1)}=\omega_{O(1)}=0, \quad u_{i O(1)}=\delta_{i 1}, \\
\phi_{O(1)}=2 \zeta_{1}=2\left(\zeta_{r} \cos \theta-\zeta_{\theta} \sin \theta\right) .
\end{gathered}
$$


$\operatorname{Order} \epsilon^{2}$ :

$$
\begin{aligned}
& P_{O(2)}=\tau_{O(2)}=\omega_{O(2)}=0 \\
& u_{r O(2)}=\frac{\gamma_{1} k c_{1}}{2 \eta^{2}}\left\{-1+\left[1+\frac{\eta}{\gamma_{1} k}(1+\cos \theta)\right] \exp \left(-\frac{\eta}{\gamma_{1} k}(1-\cos \theta)\right)\right\}, \\
& u_{\theta O(2)}=-c_{1} \frac{\sin \theta}{2 \eta} \exp \left(-\frac{\eta}{\gamma_{1} k}(1-\cos \theta)\right), \\
& u_{\varphi O(2)}=0, \\
& \phi_{O(2)}=2 \zeta_{r} u_{r O(2)}+2 \zeta_{\theta} u_{\theta O(2)}+2 \zeta_{1}^{2}-1,
\end{aligned}
$$

where $\zeta_{1}=\zeta_{r} \cos \theta-\zeta_{\theta} \sin \theta, \eta=r / \epsilon$ is a coordinate describing the slow variation in $r$ (or in $x_{i}$ ), $c_{1}$ is a constant that depends on $k$, and $\gamma_{1}$ is a dimensionless viscosity, whose definition is given in Appendix A. The $c_{1}$ will be determined later. Equations (10) and (11) show that the leading-order outer solution is the linearized uniform flow, linearized around the equilibrium state at rest. On the other hand, the velocity field given by Eq. (12) corresponds to the Oseenlet whose magnitude is modulated by the constant $c_{1}$.

To summarize, the leading-order outer solution $\phi_{O(1)}$ is given by the (linearized) uniform flow (11), while the second-order outer solution $\phi_{O(2)}$ is given by Eq. (13) with (12), provided that $c_{1}$ is known.

\section{Summary of the inner problems}

Let us now consider the solution in the near region. According to Ref. [14], the problems to determine $\phi_{I(1)}$ and $\phi_{I(2)}$ are given by

$$
\begin{gathered}
\zeta_{i} \frac{\partial \phi_{I(m)}}{\partial x_{i}}=\frac{1}{k} \mathcal{L}\left[\phi_{I(m)}\right]+\frac{1}{k} I_{m}, \quad(m=1,2), \\
\phi_{I(m)}=-2 \sqrt{\pi} \int_{\zeta_{r}<0} \zeta_{r} \phi_{I(m)} E d \zeta, \quad \zeta_{r}>0, \quad(r=1), \\
\phi_{I(m)} \rightarrow g_{m}, \quad(r \rightarrow \infty),
\end{gathered}
$$

where

$$
\begin{aligned}
& I_{1}=0, \\
& I_{2}=\mathcal{J}\left[\phi_{I(1)}, \phi_{I(1)}\right],
\end{aligned}
$$

$$
\begin{aligned}
& g_{1}=2 \zeta_{1}, \\
& g_{2}=\frac{c_{1}}{2 \gamma_{1} k}\left[-(1-\cos \theta)(1+3 \cos \theta) \zeta_{r}+2 \sin \theta(1-\cos \theta) \zeta_{\theta}\right]+2 \zeta_{1}^{2}-1,
\end{aligned}
$$

and $c_{1}$ in Eq. (18b) is the same constant as that appears in Eqs. (12b) and (12c); it will be specified later.

Equations (14)-(16) form boundary-value problems of the linearized Boltzmann equation for $\phi_{I(m)}$. In particular, the problem for $\phi_{I(1)}$ is nothing but the linearized problem for a uniform flow past a sphere, which has been investigated in the existing literature, e.g., Refs. [4,5,8-11,19,20]. For the problem with $m=2$, an inhomogeneous term appears in the equation. 


\section{SATOSHI TAGUCHI AND TOSHIHIRO SUZUKI}

For these problems, one can seek the solution in the following form [14,21]:

$$
\begin{aligned}
\phi_{I(1)}= & \varphi_{1 a}\left(r, \zeta_{r}, \zeta\right) \cos \theta+\zeta_{\theta} \varphi_{1 b}\left(r, \zeta_{r}, \zeta\right) \sin \theta, \\
\phi_{I(2)}= & -\frac{c_{1}}{2 \gamma_{1} k} \phi_{I(1)}+\varphi_{2 a}\left(r, \zeta_{r}, \zeta\right) \cos ^{2} \theta+\zeta_{\theta} \varphi_{2 b}\left(r, \zeta_{r}, \zeta\right) \cos \theta \sin \theta \\
& +\frac{\zeta_{\theta}^{2}-\zeta_{\varphi}^{2}}{2} \varphi_{2 c}\left(r, \zeta_{r}, \zeta\right) \sin ^{2} \theta+\varphi_{2 d}\left(r, \zeta_{r}, \zeta\right),
\end{aligned}
$$

where $\zeta=|\zeta|=\left(\zeta_{j}^{2}\right)^{1 / 2}$. Then, owing to the axial symmetry of the collision integral, $\mathcal{L}\left[\phi_{I(1)}\right]$, $\mathcal{L}\left[\phi_{I(2)}\right]$, and $\mathcal{J}\left[\phi_{I(1)}, \phi_{I(1)}\right]$ in the equations are decomposed into

$$
\begin{aligned}
\mathcal{L}\left[\phi_{I(1)}\right]= & \mathcal{L}\left[\varphi_{1 a}\right] \cos \theta+\zeta_{\theta} \mathcal{L}_{1}\left[\varphi_{1 b}\right] \sin \theta, \\
\mathcal{L}\left[\phi_{I(2)}\right]= & -\frac{c_{1}}{2 \gamma_{1} k} \mathcal{L}\left[\phi_{I(1)}\right]+\mathcal{L}\left[\varphi_{2 a}\right] \cos ^{2} \theta+\zeta_{\theta} \mathcal{L}_{1}\left[\varphi_{2 b}\right] \cos \theta \sin \theta \\
& +\frac{\zeta_{\theta}^{2}-\zeta_{\varphi}^{2}}{2} \mathcal{L}_{2}\left[\varphi_{2 c}\right] \sin ^{2} \theta+\mathcal{L}\left[\varphi_{2 d}\right], \\
\mathcal{J}\left[\phi_{I(1)}, \phi_{I(1)}\right]= & \mathcal{J}\left[\varphi_{1 a}, \varphi_{1 a}\right] \cos ^{2} \theta+2 \zeta_{\theta} \mathcal{J}_{1}\left[\varphi_{1 a}, \varphi_{1 b}\right] \cos \theta \sin \theta \\
& +\left(\zeta_{\theta}^{2} \mathcal{J}_{2}\left[\varphi_{1 b}, \varphi_{1 b}\right]+\mathcal{J}_{3}\left[\varphi_{1 b}, \varphi_{1 b}\right]\right) \sin ^{2} \theta,
\end{aligned}
$$

where $\mathcal{L}$ 's and $\mathcal{J}$ 's on the right-hand sides are functions of $\left(r, \zeta_{r}, \zeta\right)$. Thus, the problems for $\phi_{I(1)}$ and $\phi_{I(2)}$ are reduced to the following spatially one-dimensional problems for $\left(\varphi_{1 a}, \varphi_{1 b}\right)$ and for $\left(\varphi_{2 a}, \varphi_{2 b}, \varphi_{2 c}, \varphi_{2 d}\right)$ :

Order $\epsilon$ :

$$
\begin{aligned}
\mathcal{D} \varphi_{1 a}+\frac{\zeta^{2}-\zeta_{r}^{2}}{r} \varphi_{1 b} & =\frac{1}{k} \mathcal{L}\left[\varphi_{1 a}\right], \\
\mathcal{D} \varphi_{1 b}-\frac{\zeta_{r}}{r} \varphi_{1 b}-\frac{\varphi_{1 a}}{r} & =\frac{1}{k} \mathcal{L}_{1}\left[\varphi_{1 b}\right], \\
{\left[\begin{array}{l}
\varphi_{1 a} \\
\varphi_{1 b}
\end{array}\right] } & =\left[\begin{array}{c}
-2 \sqrt{\pi} \int_{\zeta_{r}<0} \zeta_{r} \varphi_{1 a} E d \zeta \\
0
\end{array}\right], \quad \zeta_{r}>0, \quad(r=1), \\
{\left[\begin{array}{l}
\varphi_{1 a} \\
\varphi_{1 b}
\end{array}\right] } & \rightarrow\left[\begin{array}{c}
2 \zeta_{r} \\
-2
\end{array}\right], \quad(r \rightarrow \infty) .
\end{aligned}
$$

Order $\epsilon^{2}$ :

$$
\begin{aligned}
\mathcal{D} \varphi_{2 a}+\frac{3}{2} \frac{\zeta^{2}-\zeta_{r}^{2}}{r} \varphi_{2 b} & =\frac{1}{k} \mathcal{L}\left[\varphi_{2 a}\right]+\frac{1}{k} I_{2 a}, \\
\mathcal{D} \varphi_{2 b}-\frac{\zeta_{r}}{r} \varphi_{2 b}-\frac{2}{r} \varphi_{2 a}+\frac{\zeta^{2}-\zeta_{r}^{2}}{r} \varphi_{2 c} & =\frac{1}{k} \mathcal{L}_{1}\left[\varphi_{2 b}\right]+\frac{1}{k} I_{2 b}, \\
\mathcal{D} \varphi_{2 c}-2 \frac{\zeta_{r}}{r} \varphi_{2 c}-\frac{\varphi_{2 b}}{r} & =\frac{1}{k} \mathcal{L}_{2}\left[\varphi_{2 c}\right]+\frac{1}{k} I_{2 c}, \\
\mathcal{D} \varphi_{2 d}-\frac{\zeta^{2}-\zeta_{r}^{2}}{2 r} \varphi_{2 b} & =\frac{1}{k} \mathcal{L}\left[\varphi_{2 d}\right]+\frac{1}{k} I_{2 d},
\end{aligned}
$$


ASYMPTOTIC FAR-FIELD BEHAVIOR OF MACROSCOPIC ...

$$
\begin{aligned}
& {\left[\begin{array}{l}
\varphi_{2 a} \\
\varphi_{2 b} \\
\varphi_{2 c} \\
\varphi_{2 d}
\end{array}\right]=\left[\begin{array}{c}
-2 \sqrt{\pi} \int_{\zeta_{r}<0} \zeta_{r} \varphi_{2 a} E d \zeta \\
0 \\
0 \\
-2 \sqrt{\pi} \int_{\zeta_{r}<0} \zeta_{r} \varphi_{2 d} E d \zeta
\end{array}\right], \quad \zeta_{r}>0, \quad(r=1),} \\
& {\left[\begin{array}{c}
\varphi_{2 a} \\
\varphi_{2 b} \\
\varphi_{2 c} \\
\varphi_{2 d}
\end{array}\right] \rightarrow\left[\begin{array}{c}
\frac{3}{2} \frac{c_{1}}{\gamma_{1} k} \zeta_{r}+3 \zeta_{r}^{2}-\zeta^{2} \\
-\frac{c_{1}}{\gamma_{1} k}-4 \zeta_{r} \\
2 \\
-\frac{c_{1}}{2 \gamma_{1} k} \zeta_{r}+\zeta^{2}-\zeta_{r}^{2}-1
\end{array}\right], \quad(r \rightarrow \infty) .}
\end{aligned}
$$

Here,

$$
\begin{gathered}
\mathcal{D} \varphi=\zeta_{r} \frac{\partial \varphi}{\partial r}+\frac{\zeta^{2}-\zeta_{r}^{2}}{r} \frac{\partial \varphi}{\partial \zeta_{r}} \\
{\left[\begin{array}{c}
I_{2 a} \\
I_{2 b} \\
I_{2 c} \\
I_{2 d}
\end{array}\right]=\left[\begin{array}{c}
\mathcal{J}\left[\varphi_{1 a}, \varphi_{1 a}\right]-\frac{\zeta^{2}-\zeta_{r}^{2}}{2} \mathcal{J}_{2}\left[\varphi_{1 b}, \varphi_{1 b}\right]-\mathcal{J}_{3}\left[\varphi_{1 b}, \varphi_{1 b}\right] \\
2 \mathcal{J}_{1}\left[\varphi_{1 a}, \varphi_{1 b}\right] \\
\mathcal{J}_{2}\left[\varphi_{1 b}, \varphi_{1 b}\right] \\
\frac{\zeta^{2}-\zeta_{r}^{2}}{2} \mathcal{J}_{2}\left[\varphi_{1 b}, \varphi_{1 b}\right]+\mathcal{J}_{3}\left[\varphi_{1 b}, \varphi_{1 b}\right]
\end{array}\right]}
\end{gathered}
$$

For later convenience, we introduce the following integrals with respect to the molecular velocity:

$$
\begin{aligned}
\widetilde{\omega}[\varphi] & =\langle\varphi\rangle, \quad \widetilde{u}_{r}[\varphi]=\left\langle\zeta_{r} \varphi\right\rangle, \quad \widetilde{u}_{t}[\varphi]=\frac{1}{2}\left\langle\left(\zeta^{2}-\zeta_{r}^{2}\right) \varphi\right\rangle, \\
\tilde{\tau}[\varphi] & =\frac{2}{3}\left\langle\left(\zeta^{2}-\frac{3}{2}\right) \varphi\right\rangle, \quad \widetilde{P}[\varphi]=\frac{2}{3}\left\langle\zeta^{2} \varphi\right\rangle\left(=\widetilde{\omega}[\varphi]+\widetilde{\tau}[\varphi]=\frac{\widetilde{P}_{r r}[\varphi]+2 \widetilde{P}_{t t}[\varphi]}{3}\right), \\
\widetilde{P}_{r r}[\varphi] & =2\left\langle\zeta_{r}^{2} \varphi\right\rangle, \quad \widetilde{P}_{r t}[\varphi]=\left\langle\zeta_{r}\left(\zeta^{2}-\zeta_{r}^{2}\right) \varphi\right\rangle, \quad \widetilde{P}_{t t}[\varphi]=\left\langle\left(\zeta^{2}-\zeta_{r}^{2}\right) \varphi\right\rangle, \\
\widetilde{Q}_{r}[\varphi] & =\left\langle\zeta_{r}\left(\zeta^{2}-\frac{5}{2}\right) \varphi\right\rangle, \quad \widetilde{Q}_{t}[\varphi]=\frac{1}{2}\left\langle\left(\zeta^{2}-\zeta_{r}^{2}\right)\left(\zeta^{2}-\frac{5}{2}\right) \varphi\right\rangle,
\end{aligned}
$$

where $\varphi$ is a function of $\left(\zeta_{r}, \zeta\right)$. Then, the macroscopic quantities of the inner solution up to the second order of $\epsilon$ are expressed in terms of $\left(\varphi_{1 a}, \varphi_{1 b}\right)$ and $\left(\varphi_{2 a}, \varphi_{2 b}, \varphi_{2 c}, \varphi_{2 d}\right)$ as follows:

Order $\epsilon$ :

$$
\begin{aligned}
\omega_{I(1)} & =\widetilde{\omega}_{(1)} \cos \theta, \quad u_{r I(1)}=\widetilde{u}_{r(1)} \cos \theta, \quad \tau_{I(1)}=\widetilde{\tau}_{(1)} \cos \theta, \quad P_{I(1)}=\widetilde{P}_{(1)} \cos \theta, \\
P_{r r I(1)} & =\widetilde{P}_{r r(1)} \cos \theta, \quad P_{\theta \theta I(1)}=P_{\varphi \varphi I(1)}=\widetilde{P}_{t t(1)} \cos \theta, \quad Q_{r I(1)}=\widetilde{Q}_{r(1)} \cos \theta, \\
u_{\theta I(1)} & =\widetilde{u}_{t(1)} \sin \theta, \quad P_{r \theta I(1)}=\widetilde{P}_{r t(1)} \sin \theta, \quad Q_{\theta I(1)}=\widetilde{Q}_{t(1)} \sin \theta, \\
u_{\varphi I(1)} & =P_{r \varphi I(1)}=P_{\theta \varphi I(1)}=Q_{\varphi I(1)}=0,
\end{aligned}
$$

where

$$
\begin{aligned}
\widetilde{\omega}_{(1)} & =\widetilde{\omega}\left[\varphi_{1 a}\right], \quad \widetilde{u}_{r(1)}=\widetilde{u}_{r}\left[\varphi_{1 a}\right], \quad \widetilde{\tau}_{(1)}=\widetilde{\tau}\left[\varphi_{1 a}\right], \quad \widetilde{P}_{(1)}=\widetilde{P}\left[\varphi_{1 a}\right], \\
\widetilde{P}_{r r(1)} & =\widetilde{P}_{r r}\left[\varphi_{1 a}\right], \quad \widetilde{P}_{t t(1)}=\widetilde{P}_{t t}\left[\varphi_{1 a}\right], \quad \widetilde{Q}_{r(1)}=\widetilde{Q}_{r}\left[\varphi_{1 a}\right], \\
\widetilde{u}_{t(1)} & =\widetilde{u}_{t}\left[\varphi_{1 b}\right], \quad \widetilde{P}_{r t(1)}=\widetilde{P}_{r t}\left[\varphi_{1 b}\right], \quad \widetilde{Q}_{t(1)}=\widetilde{Q}_{t}\left[\varphi_{1 b}\right] .
\end{aligned}
$$




\section{SATOSHI TAGUCHI AND TOSHIHIRO SUZUKI}

\section{$\operatorname{Order} \epsilon^{2}$ :}

$$
\begin{aligned}
& \omega_{I(2)}=-\frac{c_{1}}{2 \gamma_{1} k} \widetilde{\omega}_{(1)} \cos \theta+\widetilde{\omega}\left[\varphi_{2 a}\right] \cos ^{2} \theta+\widetilde{\omega}\left[\varphi_{2 d}\right], \\
& u_{r I(2)}=-\frac{c_{1}}{2 \gamma_{1} k} \tilde{u}_{r(1)} \cos \theta+\left(\tilde{u}_{r}\left[\varphi_{2 a}\right]-\widetilde{\omega}_{(1)} \tilde{u}_{r(1)}\right) \cos ^{2} \theta+\tilde{u}_{r}\left[\varphi_{2 d}\right], \\
& u_{\theta I(2)}=-\frac{c_{1}}{2 \gamma_{1} k} \widetilde{u}_{t(1)} \sin \theta+\left(\widetilde{u}_{t}\left[\varphi_{2 b}\right]-\widetilde{\omega}_{(1)} \widetilde{u}_{t(1)}\right) \cos \theta \sin \theta, \\
& \tau_{I(2)}=-\frac{c_{1}}{2 \gamma_{1} k} \widetilde{\tau}_{(1)} \cos \theta+\left(\tilde{\tau}\left[\varphi_{2 a}\right]-\frac{2}{3} \widetilde{u}_{r(1)}^{2}-\widetilde{\omega}_{(1)} \widetilde{\tau}_{(1)}\right) \cos ^{2} \theta \\
& +\widetilde{\tau}\left[\varphi_{2 d}\right]-\frac{2}{3} \widetilde{u}_{t(1)}^{2} \sin ^{2} \theta, \\
& P_{I(2)}=-\frac{c_{1}}{2 \gamma_{1} k} \widetilde{P}_{(1)} \cos \theta+\left(\widetilde{P}\left[\varphi_{2 a}\right]-\frac{2}{3} \widetilde{u}_{r(1)}^{2}\right) \cos ^{2} \theta+\widetilde{P}\left[\varphi_{2 d}\right]-\frac{2}{3} \widetilde{u}_{t(1)}^{2} \sin ^{2} \theta, \\
& P_{r r I(2)}=-\frac{c_{1}}{2 \gamma_{1} k} \widetilde{P}_{r r(1)} \cos \theta+\left(\widetilde{P}_{r r}\left[\varphi_{2 a}\right]-2 \widetilde{u}_{r(1)}^{2}\right) \cos ^{2} \theta+\widetilde{P}_{r r}\left[\varphi_{2 d}\right], \\
& P_{r \theta I(2)}=-\frac{c_{1}}{2 \gamma_{1} k} \widetilde{P}_{r t(1)} \sin \theta+\left(\widetilde{P}_{r t}\left[\varphi_{2 b}\right]-2 \widetilde{u}_{r(1)} \widetilde{u}_{t(1)}\right) \cos \theta \sin \theta, \\
& P_{\theta \theta I(2)}=-\frac{c_{1}}{2 \gamma_{1} k} \widetilde{P}_{t t(1)} \cos \theta+\widetilde{P}_{t t}\left[\varphi_{2 a}\right] \cos ^{2} \theta+\left\{\frac{1}{4} \widetilde{P}_{t t}\left[\left(\zeta^{2}-\zeta_{r}^{2}\right) \varphi_{2 c}\right]-2 \widetilde{u}_{t(1)}^{2}\right\} \sin ^{2} \theta+\widetilde{P}_{t t}\left[\varphi_{2 d}\right], \\
& P_{\varphi \varphi I(2)}=-\frac{c_{1}}{2 \gamma_{1} k} \widetilde{P}_{t t(1)} \cos \theta+\widetilde{P}_{t t}\left[\varphi_{2 a}\right] \cos ^{2} \theta-\frac{1}{4} \widetilde{P}_{t t}\left[\left(\zeta^{2}-\zeta_{r}^{2}\right) \varphi_{2 c}\right] \sin ^{2} \theta+\widetilde{P}_{t t}\left[\varphi_{2 d}\right] \\
& Q_{r I(2)}=-\frac{c_{1}}{2 \gamma_{1} k} \widetilde{Q}_{r(1)} \cos \theta+\left(\widetilde{Q}_{r}\left[\varphi_{2 a}\right]+\frac{5}{2} \widetilde{\omega}_{(1)} \widetilde{u}_{r(1)}-\widetilde{u}_{r(1)} \widetilde{P}_{r r(1)}\right. \\
& \left.-\frac{3}{2} \widetilde{P}_{(1)} \widetilde{u}_{r(1)}\right) \cos ^{2} \theta+\widetilde{Q}_{r}\left[\varphi_{2 d}\right]-\widetilde{u}_{t(1)} \widetilde{P}_{r t(1)} \sin ^{2} \theta, \\
& Q_{\theta I(2)}=-\frac{c_{1}}{2 \gamma_{1} k} \widetilde{Q}_{t(1)} \sin \theta+\left(\widetilde{Q}_{t}\left[\varphi_{2 b}\right]+\frac{5}{2} \widetilde{\omega}_{(1)} \widetilde{u}_{t(1)}-\widetilde{u}_{r(1)} \widetilde{P}_{r t(1)}\right. \\
& \left.-\widetilde{u}_{t(1)} \widetilde{P}_{t t(1)}-\frac{3}{2} \widetilde{P}_{(1)} \widetilde{u}_{t(1)}\right) \cos \theta \sin \theta, \\
& u_{\varphi I(2)}=P_{r \varphi I(2)}=P_{\theta \varphi I(2)}=Q_{\varphi I(2)}=0 .
\end{aligned}
$$

Note that $\widetilde{\omega}[\cdot], \widetilde{u}_{r}[\cdot], \widetilde{u}_{t}[\cdot]$, etc., in Eq. (28) as well as $\widetilde{\omega}_{(1)}, \widetilde{u}_{r(1)}, \widetilde{u}_{t(1)}$, etc. in Eqs. (26) and (28) are functions of $r$ only.

To summarize, the leading-order inner solution is obtained by solving the problem (21a)-(21d), while the second-order inner solution by solving the problem (22a)-(22f), provided that the constant $c_{1}$ is known. Once the solutions are known, the macroscopic quantities are given by Eqs. (26) and (28).

\section{Asymptotic behavior of the leading-order inner solution at large $r \gg 1$}

Up to now, we have left the constant $c_{1}$ undetermined. The $c_{1}$ appears in the expression of $\phi_{O(2)}$ as well as in the problem for $\phi_{I(2)}$, namely, at the second order level. In this section, we explain how it is determined.

For this purpose, let us consider the asymptotic behavior of the leading-order inner solution $\phi_{I(1)}$ at large $r \gg 1$; it can be investigated [11,14] with the aid of the asymptotic theory of the 
Boltzmann equation [22,23]. First, the moments $\widetilde{\omega}_{(1)}, \widetilde{u}_{r(1)}, \widetilde{\tau}_{(1)}, \widetilde{P}_{(1)}$, and $\widetilde{u}_{t(1)}$ of $\varphi_{1 a}$ and $\varphi_{1 b}$ at large $r$ are expressed as

$$
\begin{aligned}
& \tilde{u}_{r(1)}(r)=1+\frac{c_{1}}{r}+\frac{c_{2}}{r^{3}}, \quad \tilde{u}_{t(1)}(r)=-1-\frac{c_{1}}{2 r}+\frac{c_{2}}{2 r^{3}}, \\
& \widetilde{P}_{(1)}(r)=\gamma_{1} k \frac{c_{1}}{r^{2}}, \quad \widetilde{\tau}_{(1)}(r)=\frac{c_{3}}{r^{2}}, \quad \widetilde{\omega}_{(1)}(r)=\frac{\gamma_{1} k c_{1}-c_{3}}{r^{2}},
\end{aligned}
$$

where $c_{i}(i=1,2,3)$ are constants. The forms are derived from the fact that the flow velocity and pressure fields far from the sphere are described by the Stokes equation for an incompressible fluid, while the temperature field is described by the Laplace equation; the constants $c_{1}, c_{2}$, and $c_{3}$ were introduced because the boundary condition on the sphere surface is not specified. The corresponding VDF at large $r$ is also given using $c_{i}$ as

$$
\begin{aligned}
\varphi_{1 a}= & 2\left(1+\frac{c_{1}}{r}+\frac{c_{2}}{r^{3}}\right) \zeta_{r}+\frac{c_{3}}{r^{2}}\left(\zeta^{2}-\frac{5}{2}\right) \\
& +\frac{k}{r^{2}}\left[\gamma_{1} c_{1}+\frac{2 c_{3}}{r} \zeta_{r} A(\zeta)-\frac{1}{2}\left(c_{1}+\frac{3 c_{2}}{r^{2}}\right) B(\zeta)\left(\zeta^{2}-3 \zeta_{r}^{2}\right)\right] \\
& -\frac{k^{2}}{r^{3}}\left\{2 c_{1} \zeta_{r} D_{1}(\zeta)-\frac{3 c_{3}}{r} F(\zeta)\left(\zeta^{2}-3 \zeta_{r}^{2}\right)\right. \\
& \left.+2\left[c_{1}\left(2 \zeta^{2}-3 \zeta_{r}^{2}\right)+\frac{3 c_{2}}{r^{2}}\left(3 \zeta^{2}-5 \zeta_{r}^{2}\right)\right] \zeta_{r} D_{2}(\zeta)\right\}+\cdots, \\
\varphi_{1 b}= & -2-\frac{c_{1}}{r}+\frac{c_{2}}{r^{3}}+\frac{k}{r^{3}}\left[c_{3} A(\zeta)+\frac{3 c_{2}}{r} \zeta_{r} B(\zeta)\right] \\
& -\frac{k^{2}}{r^{3}}\left\{c_{1} D_{1}(\zeta)+\frac{6 c_{3}}{r} \zeta_{r} F(\zeta)\right. \\
& \left.+\frac{1}{2}\left[c_{1}\left(\zeta^{2}-3 \zeta_{r}^{2}\right)+\frac{9 c_{2}}{r^{2}}\left(\zeta^{2}-5 \zeta_{r}^{2}\right)\right] D_{2}(\zeta)\right\}+\cdots,
\end{aligned}
$$

where $A(\zeta), B(\zeta)$, etc. are the solutions of the integral equations related to the linearized collision integral, whose definitions are summarized in Appendix A. Thus, $c_{1}$ is the factor of the most slowly decaying terms $\left(r^{-1}\right.$ terms) in $\phi_{I(1)}$ (and in the flow velocity). Similarly, $c_{3}$ and $c_{2}$ correspond to the factors of the second and third most slowly decaying terms in VDF, respectively. They are determined together with the solution.

The $c_{1}$ thus introduced in the leading-order solution is then inherited in the second-order solutions through the matching procedure [14].

To summarize, the process of the asymptotic analysis up to order $\epsilon^{2}$ is as follows: (i) We first solve the first-order inner problem (21a)-(21d) to obtain $\phi_{I(1)}$ and any macroscopic quantity of our interest at order $\epsilon$ from Eqs. (26) and (27). From the asymptotic behavior at large $r \gg 1, c_{1}$ is obtained (and $c_{2}$ and $c_{3}$, if necessary). (ii) With $c_{1}$ thus obtained, the second-order outer solution $\phi_{O(2)}$ and the problem to determine the second-order inner solution $\phi_{I(2)}$ are determined; they are given by Eq. (13) with (12) and by Eqs. (22a)-(22f), respectively. (iii) Solving the problem for $\phi_{I(2)}$ thus obtained, the behavior of the gas near the sphere is determined at the second order of $\epsilon$.

It should be noted that the information on $c_{2}$ and $c_{3}$ is not required as far as the solution up to the order $\epsilon^{2}$ is concerned. Moreover, as far as the net force acting on the sphere (drag) is concerned, one can skip the process (iii) as described below. 


\section{SATOSHI TAGUCHI AND TOSHIHIRO SUZUKI}

For later convenience, we arrange Eq. (29) to obtain

$$
\begin{aligned}
& c_{1}=\frac{1}{2} \lim _{r \rightarrow \infty} r\left(\tilde{u}_{r(1)}-2 \tilde{u}_{t(1)}-3\right), \\
& c_{2}=\frac{1}{2} \lim _{r \rightarrow \infty} r^{3}\left(\widetilde{u}_{r(1)}+2 \widetilde{u}_{t(1)}+1\right), \\
& c_{3}=\lim _{r \rightarrow \infty} r^{2} \widetilde{\tau}_{(1)} .
\end{aligned}
$$

\section{Drag acting on the sphere}

Now we consider the net force acting on the sphere. We consider here a slightly more general case in which the upstream flow velocity is given by $v_{\infty i}$ instead of $\left(v_{\infty}, 0,0\right)$. Let us denote by $F_{i}$ the force exerted on the sphere by the uniform flow $v_{\infty i}$. Obviously, $F_{i}$ is obtained from that for the present case of $v_{\infty i}=v_{\infty} \delta_{i 1}$ by the rotation of the coordinate axes, and the latter is obtained by integrating the momentum flux over the surface of the sphere, whose angular dependency is explicit owing to Eqs. (26) and (28). Thus, we obtain, after omitting the terms smaller than $O\left(\epsilon^{2}\right)$,

$$
\begin{gathered}
F_{i}=p_{\infty} L^{2}\left(2 R T_{\infty}\right)^{-1 / 2} v_{\infty i}\left[1-\frac{c_{1}(k)}{2 \gamma_{1} k} \epsilon\right] h_{D}(k), \\
h_{D}=-\frac{4}{3} \pi\left[\widetilde{P}_{r r(1)}(1)-2 \widetilde{P}_{r t(1)}(1)\right],
\end{gathered}
$$

where $\epsilon=\left|v_{\infty i}\right| /\left(2 R T_{\infty}\right)^{1 / 2}$ with $\left|v_{\infty i}\right|=\left(v_{\infty j}^{2}\right)^{1 / 2}, \widetilde{P}_{r r(1)}(r)$ and $\widetilde{P}_{r t(1)}(r)$ depending on $r$ are defined in Eq. (27), and the meaning of the argument $k$ for $c_{1}$ and $h_{D}$ in Eq. (32) is explained below.

It should be noted that $c_{1}$ and $h_{D}$ are derived from the leading-order inner solution $\phi_{I(1)}$, which contains the parameter $k$. Thus, $c_{1}$ and $h_{D}$ also depend on $k$ through $\phi_{I(1)}$. Therefore, if $c_{1}$ and $h_{D}$ are regarded as functions of $k$, they provide the information on the effect of gas rarefaction in the drag formula (32). In this way, our task of constructing the second order drag is reduced to the task of constructing two functions $h_{D}$ and $c_{1}$ of the Knudsen number, by solving the leading-order inner problem for various $k$. This striking simplification, pointed out in Ref. [14], is one of important outcomes of the asymptotic analysis.

Before leaving this section, we summarize here some results for small $k$. The asymptotic forms of $h_{D}$ and $c_{1}$ for small $k$ can be obtained with the aid of the asymptotic theory of the Boltzmann equation [22,23]. They are summarized as

$$
\begin{gathered}
h_{D}=6 \pi \gamma_{1} k\left(1-b_{1}^{(1)} k+a_{D} k^{2}+\cdots\right), \\
c_{1}=-\frac{3}{2}\left(1-b_{1}^{(1)} k+a_{D} k^{2}+\cdots\right), \\
c_{2}=\frac{1}{2}\left(1-3 b_{1}^{(1)} k+3 b_{D} k^{2}+\cdots\right), \\
c_{3}=6 c_{3}^{(0)} k^{2}+\cdots .
\end{gathered}
$$

Here, the results for $c_{2}$ and $c_{3}$, also depending on $k$, have been included. The $b_{1}^{(1)}$ and $c_{3}^{(0)}$ are the so-called slip or jump coefficients and $a_{D}$ and $b_{D}$ are given by

$$
\begin{aligned}
& a_{D}=3\left(b_{1}^{(1)}\right)^{2}+4 b_{4}^{(1)}-b_{5}^{(1)}-b_{6}^{(1)}-\int_{0}^{\infty} Y_{1}^{(1)}(z) d z \\
& b_{D}=3\left(b_{1}^{(1)}\right)^{2}+4 b_{4}^{(1)}-b_{5}^{(1)}-b_{6}^{(1)}+\int_{0}^{\infty} Y_{1}^{(1)}(z) d z
\end{aligned}
$$

where $b_{i}^{(1)}(i=4,5,6)$ are also slip coefficients and $Y_{1}^{(1)}$ is a Knudsen-layer function. The formula for $h_{D}$ was taken from Ref. [22], which was originally obtained in Ref. [5] for the BGK model. The numerical values of the slip or jump coefficients are listed in Table I for the hard-sphere gas 
TABLE I. Slip or jump coefficients occurring in Eqs. (34)-(38) for the hard-sphere (HS) gas, the ES model $(\operatorname{Pr}=2 / 3)$, and the BGK model (or the ES model with $\operatorname{Pr}=1)$ under the diffuse reflection condition. Data are taken from http://hdl.handle.net/2433/199811 (see also Ref. [24]). The values of $a_{D}$ and $b_{D}$ are also included.

\begin{tabular}{lcrrr}
\hline \hline Present notation & Notation in Ref. [22] & HS & ES & BGK \\
\hline$b_{1}^{(1)}$ & $-k_{0}$ & 1.25395 & 0.67746 & 1.01619 \\
$b_{4}^{(1)}$ & $-a_{1}$ & -0.90393 & -0.51088 & -0.76632 \\
$b_{5}^{(1)}$ & $-a_{2}$ & -0.66012 & -0.33334 & -0.50000 \\
$b_{6}^{(1)}$ & $-a_{3}$ & 0.24381 & 0.17753 & 0.26632 \\
$\int_{0}^{\infty} Y_{1}^{(1)} d z$ & $-2 b_{1}$ & -0.21369 & -0.15578 & -0.23368 \\
$c_{3}^{(0)}$ & $d_{4}$ & 0.00874 & 0.00041 & 0.11169 \\
$a_{D}$ & $-(4 / 3) A_{\mathrm{d}}$ & 1.73145 & -0.35507 & 0.50001 \\
$b_{D}$ & $(4 / 3) B_{\mathrm{d}}$ & 1.30407 & -0.66663 & 0.03265 \\
\hline \hline
\end{tabular}

as well as for the ES model ( $\mathrm{Pr}=2 / 3)$, including the case of the BGK model, under the diffuse reflection boundary condition. It may be mentioned that the complete numerical data including the second-order slip or jump coefficients have been obtained recently for the hard-sphere gas and for the ES model [24-26] (available at Ref. [27] in a convenient form). We followed the notations introduced in these references and indicated the correspondence to those of Ref. [22] in the table. We also remark that although $k$ is assumed to be finite in our analysis, the result of the asymptotic analysis is valid also for small $k$ as long as $\epsilon$ is sufficiently small [14].

\section{CONSTRUCTION OF FUNCTIONS $c_{1}$ AND $c_{3}$}

In the preceding section, we have shown that the second-order drag is obtained by constructing the function $c_{1}(k)$ in addition to $h_{D}(k)$, both of which are obtained from the leading-order inner problem. The main difficulty in computing $c_{1}$, as compared to $h_{D}$, is that it is a quantity related to the decay of the solution at large $r$, which is not practical to obtain numerically. Therefore, in this section, we first derive the relation between $h_{D}(k)$ and $c_{1}(k)$, which allows one to obtain $c_{1}(k)$ directly from $h_{D}(k)$ once we know the latter. We will also derive a similar representation formula for $c_{3}$ occurring in the asymptotic expressions of $\widetilde{\tau}_{(1)}$ and $\widetilde{\omega}_{(1)}$ in Eq. (29b), which establishes the connection of $c_{3}$ with the thermal force in the problem of thermophoresis.

\section{A. Representation formulas for $c_{1}$ and $c_{3}$}

Let us consider a sphere centered at the origin and having radius $r>1$, thus enclosing the original unit sphere. The surface of this sphere with radius $r$ is denoted by $S_{r}$. Owing to the conservation of momentum, the momentum flux in the $x_{1}$ direction integrated over $S_{r}$ and that integrated over the unit sphere should be identical, thus yielding the equality

$$
h_{D}=-\frac{4}{3} \pi r^{2}\left[\widetilde{P}_{r r(1)}(r)-2 \widetilde{P}_{r t(1)}(r)\right],
$$

where $h_{D}$ is given by Eq. (33). Now we consider $S_{r}$ with large $r \gg 1$. Then, we can safely use the asymptotic form (30) of $\varphi_{1 a}$ and $\varphi_{1 b}$ there, from which we obtain the expressions $\widetilde{P}_{r r(1)}=$ $3 \gamma_{1} k c_{1} / r^{2}+O\left(r^{-4}\right)$ and $\widetilde{P}_{r t(1)}=O\left(r^{-4}\right)$ for $r \gg 1$. Substituting them into Eq. (39) gives $h_{D}=$ $-4 \pi \gamma_{1} k c_{1}+O\left(r^{-2}\right)$. Letting $r \rightarrow \infty$, we obtain the following representation of $c_{1}$ in terms of $h_{D}$ :

$$
c_{1}=-\frac{h_{D}}{4 \pi \gamma_{1} k} \text {. }
$$




\section{SATOSHI TAGUCHI AND TOSHIHIRO SUZUKI}

Thus, $h_{D}$ and $c_{1}$ are not independent but are related each other. This is a useful relationship because it allows us to derive $c_{1}$ from $h_{D}$, the latter of which is much easier to compute.

To derive a similar formula for $c_{3}$, let us consider the following associated problem (say, Problem T). Consider a sphere (radius $L$ and temperature $T_{0}$ ) immersed in an infinite expanse of otherwise undisturbed gas at rest with uniform pressure $p_{0}$ and temperature $T_{0}\left(1+\alpha X_{1} / L\right)$, where $\alpha$ is a dimensionless constant whose magnitude is assumed to be small $(|\alpha| \ll 1)$. The sphere center is located at the origin of the spatial coordinate system. In this situation, a force (thermal force) is exerted on the sphere as a result of the flow induced around the sphere (i.e., thermophoresis; see, e.g., Refs. [28-30] and the references therein). We investigate this problem on the basis of the linearized Boltzmann equation and the diffuse reflection boundary condition.

Let us denote by $p_{0} L^{2}\left(\hat{F}_{T}, 0,0\right)$ the force exerted on the sphere in Problem T. Then, it was shown in Ref. [31] (see Example 3 there) that $\hat{F}_{T}$ is expressed in terms of the quantities of the present problem of the uniform flow in the following forms:

$$
\begin{aligned}
\hat{F}_{T} & =\alpha \int_{r=1}\left[x_{1} Q_{r I(1)}+k\left\langle\zeta_{r} \zeta_{1} A(\zeta) \phi_{I(1)}\right\rangle\right] d S \\
& =\alpha \lim _{r \rightarrow \infty} \int_{S_{r}}\left[x_{1} Q_{r I(1)}+k\left\langle\zeta_{r} \zeta_{1} A(\zeta) \phi_{I(1)}\right\rangle\right] d S,
\end{aligned}
$$

where $d S$ is the surface element. With the aid of the similarity solution introduced earlier, the integral on the most right-hand side is simplified to give

$$
\begin{aligned}
& \int_{S_{r}} {\left[x_{1} Q_{r I(1)}+k\left\langle\zeta_{r} \zeta_{1} A(\zeta) \phi_{I(1)}\right\rangle\right] d S } \\
& \quad=\frac{4}{3} \pi r^{2}\left\{r \widetilde{Q}_{r(1)}(r)+k\left[\left\langle\zeta_{r}^{2} A(\zeta) \varphi_{1 a}\right\rangle-\left\langle\zeta_{r}\left(\zeta^{2}-\zeta_{r}^{2}\right) A(\zeta) \varphi_{1 b}\right\rangle\right]\right\}
\end{aligned}
$$

Now we examine each term on the right-hand side with the aid of the asymptotic expressions for $\varphi_{1 a}$ and $\varphi_{1 b}$ for $r \gg 1$, given in Sec. II B 4. Simple computations show

$$
\begin{aligned}
\widetilde{Q}_{r(1)}(r) & =\left(\frac{5}{2} \gamma_{2} c_{3}-\gamma_{3} k c_{1}\right) \frac{k}{r^{3}} \\
\left\langle\zeta_{r}^{2} A(\zeta) \varphi_{1 a}\right\rangle & =\left(\frac{5}{4} \gamma_{2} c_{3}+\gamma_{3} k c_{1}\right) \frac{1}{r^{2}}+O\left(r^{-4}\right), \\
\left\langle\zeta_{r}\left(\zeta^{2}-\zeta_{r}^{2}\right) A(\zeta) \varphi_{1 b}\right\rangle & =O\left(r^{-4}\right),
\end{aligned}
$$

for $r \gg 1$, where $\gamma_{3}$ is the constant defined in Appendix A. Thus, the most right-hand side of Eq. (41) is reduced to $5 \pi \alpha \gamma_{2} k c_{3}$ when $r \rightarrow \infty$. Since the second integral of Eq. (41) is evaluated on the sphere, we have the following representation formula of $c_{3}$ in terms of the quantities on the boundary:

$$
c_{3}=\frac{4}{15 \gamma_{2} k}\left\{\widetilde{Q}_{r(1)}(1)+k\left[\left\langle\left.\zeta_{r}^{2} A(\zeta) \varphi_{1 a}\right|_{r=1}\right\rangle-\left\langle\left.\zeta_{r}\left(\zeta^{2}-\zeta_{r}^{2}\right) A(\zeta) \varphi_{1 b}\right|_{r=1}\right\rangle\right]\right\}
$$

where the integral was again simplified by the use of the similarity solution. It also gives the direct connection of $c_{3}$ with the thermal force, i.e.,

$$
\hat{F}_{T}=5 \pi \alpha \gamma_{2} k c_{3}
$$

Since $c_{3}=6 c_{3}^{(0)} k^{2}+\cdots$ for small $k$, we have $\hat{F}_{T}=30 \pi \alpha \gamma_{2} c_{3}^{(0)} k^{3}+\cdots$ for $k \ll 1$, the formula which was first obtained in Ref. [30] (note the correspondence $d_{4}=c_{3}^{(0)}$ in notations). In this sense, 
TABLE II. $h_{D}(k), c_{1}(k), h_{T}(k)$, and $c_{3}(k)$ for a hard-sphere gas under the diffuse reflection boundary condition. The data for $h_{D}$ and $h_{T}$ are taken from Ref. [22], and the values of $c_{1}$ and $c_{3}$ are obtained with the aid of the formulas (40) and (44), including the values at $k=\infty$.

\begin{tabular}{lcccc}
\hline \hline$k$ & $h_{D}$ & $-c_{1}$ & $h_{T}$ & $-c_{3}$ \\
\hline 0.05 & 1.1091 & 1.38986 & -0.0068 & 0.00054 \\
0.1 & 2.1168 & 1.32633 & -0.0457 & 0.00364 \\
0.2 & 3.8110 & 1.19394 & -0.2075 & 0.01651 \\
0.4 & 6.2292 & 0.97576 & -0.6017 & 0.04788 \\
0.6 & 7.7951 & 0.81403 & -0.9034 & 0.07189 \\
1 & 9.5625 & 0.59916 & -1.2585 & 0.10015 \\
2 & 11.2772 & 0.35330 & -1.6001 & 0.12733 \\
4 & 12.2333 & 0.19163 & -1.7818 & 0.14179 \\
6 & 12.5557 & 0.13112 & -1.8399 & 0.14641 \\
10 & 12.8071 & 0.08025 & -1.8838 & 0.14991 \\
$\infty$ & 13.1653 & 0 & -1.9423 & 0.15456 \\
\hline \hline
\end{tabular}

the present result is an extension of the formula in Ref. [30] to the case of arbitrary finite Knudsen number.

For the sake of later convenience, let us introduce a slightly different normalization of the thermal force $p_{0} L^{2} \hat{F}_{T}=L^{2}\left(2 R T_{0}\right)^{-1 / 2} \lambda_{0}\left(\partial T / \partial X_{1}\right)_{\infty} h_{T}(k)$, where $\lambda_{0}$ is the thermal conductivity of the gas (see Appendix A). Here, the function $h_{T}(k)$ is of our interest in place of $\hat{F}_{T}$. With this $h_{T}$, the relation between the thermal force and $c_{3}$ is recast as

$$
h_{T}=4 \pi c_{3} .
$$

Finally, we note that the above derivations of the representation formulas rely only on the general form of the velocity distribution function, i.e., Eq. (30), and does not use any specific information of the molecular model. Thus, the relations hold for the general molecular model, not restricted to the hard-sphere gas nor the ES model. Moreover, it holds for the general (locally isotropic) boundary condition, not restricted to the diffuse reflection condition.

\section{B. Functions $c_{1}(k)$ and $c_{3}(k)$ for a hard-sphere gas}

A direct consequence of the relations (40) and (44) is that if we know $h_{D}$ and $h_{T}$, we can immediately obtain $c_{1}$ and $c_{3}$. Fortunately, this is the case for the hard-sphere gas, for which accurate numerical data of $h_{D}$ [11] and those of $h_{T}$ [29] (see also Ref. [22]) are available (under the diffuse reflection boundary condition). Therefore, we can exploit them to obtain the numerical data of $c_{1}$ and $c_{3}$ for the hard-sphere gas. The results are shown in Table II. With the data of $c_{1}$ thus obtained, the second-order drag is now available for the hard-sphere gas under the diffuse reflection condition. The similar results for the ES model as well as for the BGK model will be given in the remaining part of the paper.

\section{Functions $c_{1}(k)$ and $c_{3}(k)$ for the ES model}

In the previous section, we obtained $c_{1}$ and $c_{3}$ for the hard-sphere gas, taking full advantage of the available data and the formulas (40) and (44). Here, we construct the similar results for the ES model. Unlike the case of the hard-sphere gas, the values of $h_{D}$ and $h_{T}$ are unavailable for this model. Therefore, we first construct $h_{D}$ for the ES model by solving the boundary-value problem (21a)-(21d) numerically and then obtain $c_{1}$ by the formula (40). As for $c_{3}$, we will not solve the problem of thermophoresis to obtain $h_{T}$. Instead, we will use the representation formula (42) to 


\section{SATOSHI TAGUCHI AND TOSHIHIRO SUZUKI}

obtain $c_{3}$ from the solution of the present linearized problem, which also gives $h_{T}$ owing to the relation (44).

\section{Preliminary}

The ES model of the collision integral is well known and only the explicit form of its linearized form is summarized in Appendix A [see Eq. (A9)]. Then, the corresponding forms of $\mathcal{L}\left[\varphi_{1 a}\right]$ and $\mathcal{L}_{1}\left[\varphi_{1 b}\right]$, appearing in Eqs. (21a) and (21b), are readily obtained as

$$
\begin{aligned}
& \mathcal{L}^{\mathrm{ES}}\left[\varphi_{1 a}\right]=\widetilde{\omega}_{(1)}+2 \zeta_{r} \widetilde{u}_{r(1)}+\left(\zeta^{2}-\frac{3}{2}\right) \widetilde{\tau}_{(1)}+v\left[\zeta_{r}^{2} \widetilde{P}_{r r(1)}+\left(\zeta^{2}-\zeta_{r}^{2}\right) \widetilde{P}_{t t(1)}-\zeta^{2} \widetilde{P}_{(1)}\right]-\varphi_{1 a}, \\
& \mathcal{L}_{1}^{\mathrm{ES}}\left[\varphi_{1 b}\right]=2\left(\widetilde{u}_{t(1)}+v \zeta_{r} \widetilde{P}_{r t(1)}\right)-\varphi_{1 b},
\end{aligned}
$$

where $\widetilde{\omega}_{(1)}, \widetilde{u}_{r(1)}, \widetilde{\tau}_{(1)}, \widetilde{P}_{r r(1)}, \widetilde{P}_{t t(1)}, \widetilde{P}_{(1)}, \widetilde{u}_{t(1)}$, and $\widetilde{P}_{r t(1)}$ are given in Eq. (27) [with Eq. (25)], and $v \in[-1 / 2,1)$ is a parameter. $v$ is related to the Prandtl number Pr by the relation

$$
\operatorname{Pr}=\frac{1}{1-v} \text {. }
$$

Thus, by setting $v=-1 / 2$, the model can yield the Prandtl number $\operatorname{Pr}=2 / 3$ in the continuum flow limit, which is the value for the pseudo-Maxwell molecules [32]. The ES model also satisfies the Boltzmann H-theorem in the full nonlinear operator [33]. All these desirable properties have stimulated further investigations on the model, including its extension to gas mixtures, e.g., Refs. [34,35].

The solution method used in the present study is essentially the same as that of Ref. [11]. It is a finite-difference method with an ability to resolve the discontinuity contained in VDF. The main difference lies in the part of computing the discontinuity propagating in the gas along the characteristics of the equation, which is briefly explained in Appendix B. The computations were carried out for the canonical cases $\operatorname{Pr}=2 / 3(v=-1 / 2)$ and $\operatorname{Pr}=1(v=0)$, the latter of which corresponds to the case of the BGK model. Note that although the values of $h_{D}$ and $c_{1}$ for the BGK model were reported in Ref. [14], the present results are based on new computations, for which higher accuracy was attained.

\section{Behavior of the macroscopic quantities}

Before presenting the result for $h_{D}, c_{1}$, and $c_{3}$ for the ES model, we first show the behavior of the macroscopic quantities at large $r$. We show in Fig. 1 the double-log plots of the moments $\widetilde{\omega}_{(1)}$, $\widetilde{u}_{r(1)}-1, \tilde{u}_{t(1)}+1$, and $\widetilde{\tau}_{(1)}$ for various $k$ in the cases of $\operatorname{Pr}=2 / 3$ and 1 (or the BGK model). As seen from the figures, the density and temperature decay in proportion to $r^{-2}$, whereas the flow velocity decays in proportion to $r^{-1}$. The decay rate is consistent with Eq. (29), which is the basis of our asymptotic analysis. The result of the macroscopic quantities in the free molecular limit $(k \rightarrow \infty)$ is also included in the figure and is represented by the dashed line, though our asymptotic analysis assumes finite $k$. It is easy to check, in the collisionless case, that the radial flow velocity $\left(\widetilde{u}_{r(1)}\right)$ and the circumferential flow velocity $\left(\widetilde{u}_{t(1)}\right)$ decay in proportion to $r^{-2}$ and $r^{-3}$, respectively, which makes a clear contrast with the collisional case. It should also be pointed out that the magnitude of $\tilde{\tau}_{(1)}$ in the case of $\operatorname{Pr}=2 / 3$ is very small as compared to that in the case of $\operatorname{Pr}=1$ when $k$ is small $(k=0.1)$.

\section{Functions $h_{D}(k), c_{1}(k)$, and $c_{3}(k)$}

Now we show the results for $h_{D}$ and $c_{1}$ for the ES model. The value of $h_{D}$ is directly computed from Eq. (33). Once $h_{D}$ is known, $c_{1}$ is readily obtained with the aid of Eq. (40). The results of $h_{D}$ and $c_{1}$ thus obtained are shown in Table III for $\operatorname{Pr}=2 / 3$ and in Table IV for $\operatorname{Pr}=1$ (or the BGK model); the values of $c_{1}$ are tabulated in the column "formula (40)." $h_{D}$ takes positive values and increases monotonically with $k$, whereas $c_{1}$ is negative and its magnitude decreases in $k$. This general trend is the same as in the case of the hard-sphere gas. Since $h_{D}$ remains finite for all $k$ 
ASYMPTOTIC FAR-FIELD BEHAVIOR OF MACROSCOPIC ...

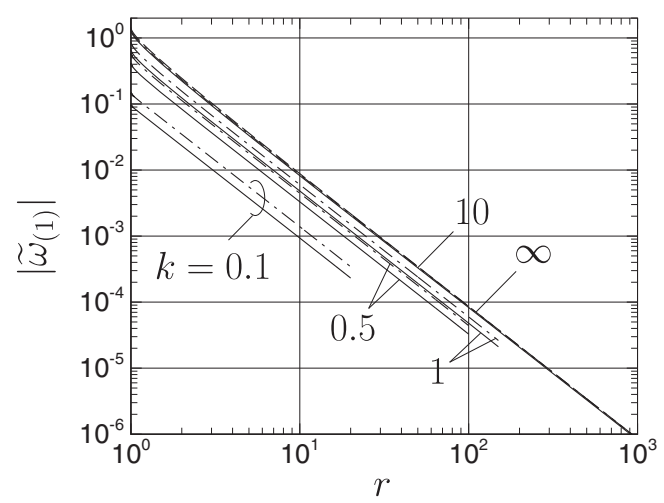

(a)

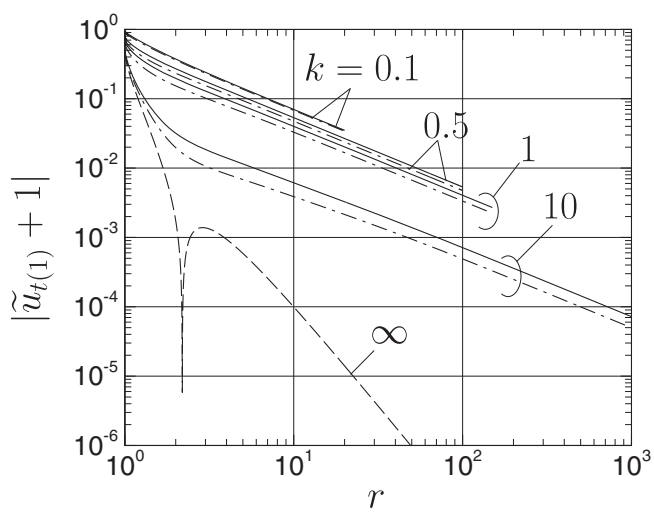

(c)



(b)

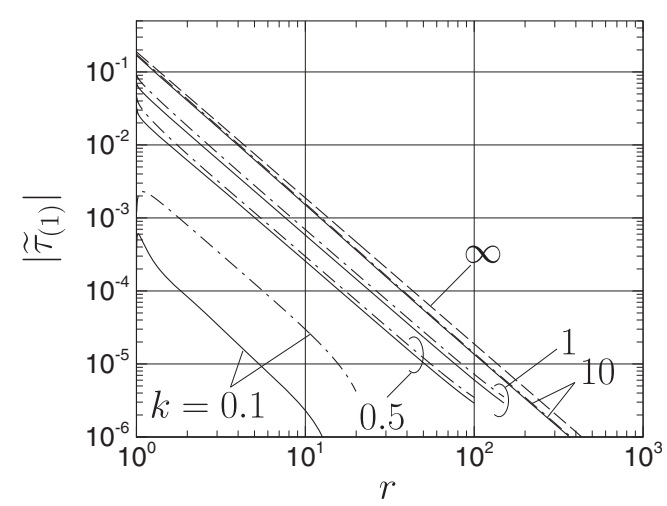

(d)

FIG. 1. Double-log plots of the moments $\widetilde{\omega}_{(1)}, \widetilde{u}_{r(1)}-1, \widetilde{u}_{t(1)}+1$, and $\widetilde{\tau}_{(1)}$ for $\operatorname{Pr}=2 / 3$ and 1 on the basis of the ES model under the diffuse reflection boundary condition. The solid line indicates the result for $\operatorname{Pr}=2 / 3$ and the dash-dot line the result for $\operatorname{Pr}=1$ (or the BGK model). The solution of the free-molecular gas $(k=\infty)$ is indicated by the dashed line.

(including the limit $k \rightarrow \infty$ ), Eq. (40) suggests $c_{1} \rightarrow 0$ in the limit $k \rightarrow \infty$. Though Eq. (40) was derived exclusively in the case of finite $k$, this conclusion is consistent with our direct numerical results presented below. With the data of $c_{1}$ thus obtained, the second-order drag is now available for the ES model $(\mathrm{Pr}=2 / 3)$ as well as for the BGK model under the diffuse reflection condition.

Next, we present the values of $c_{3}$ obtained from the numerical solutions with the aid of Eq. (42). The integrals with respect to the molecular velocity on the right-hand side were carried out numerically by Simpson's rule in the same way as in the computations of the macroscopic quantities. The results are shown in the column "formula (42)" in Table III $(\operatorname{Pr}=2 / 3)$ and in Table IV $(\operatorname{Pr}=1$, i.e., the BGK model). While $c_{3}$ is negative for moderate values of $k$, it takes positive values for small $k$. This positivity for small $k$ is also confirmed by the asymptotic expression (37), where $c_{3}^{(0)}$ is positive for the ES model (including the BGK model) as well as for the hard-sphere model. Recalling that $c_{3}$ is essentially the thermal force $h_{T}$ [Eq. (44)], this trend is in agreement with that of $h_{T}$, for which the positive $h_{T}$ is observed for small $k$ (known as the negative thermophoresis [36,37]). 


\section{SATOSHI TAGUCHI AND TOSHIHIRO SUZUKI}

TABLE III. $h_{D}, c_{1}, c_{2}$, and $c_{3}$ vs $k$ for the ES model with $\operatorname{Pr}=2 / 3$ under the diffuse reflection boundary condition. The values in the columns "formula (40)" and "formula (42)" were obtained with the aid of Eqs. (40) and (42), respectively, while those in the columns "direct computation" were obtained by the fitting method explained in the main text. The deviation of the slope of the fitting line from the theoretical value is shown in the parentheses.

\begin{tabular}{|c|c|c|c|c|c|c|c|c|c|}
\hline \multirow[b]{2}{*}{$k$} & \multirow[b]{2}{*}{$h_{D}$} & \multirow{2}{*}{$\begin{array}{l}\text { Formula (40) } \\
\qquad-c_{1}\end{array}$} & \multirow{2}{*}{$\begin{array}{c}\text { Formula (42) } \\
-c_{3}\end{array}$} & \multicolumn{6}{|c|}{ Direct computation } \\
\hline & & & & & $-c_{1}$ & & $-c_{2}$ & & $-c_{3}$ \\
\hline 0.1 & 1.1707 & 1.39742 & -0.0003 & 1.3975 & $\left(2.4 \times 10^{-6}\right)$ & -0.391 & $\left(1.4 \times 10^{-4}\right)$ & -0.0003 & $\left(8.6 \times 10^{-2}\right)$ \\
\hline 0.15 & 1.6946 & 1.34851 & 0.0006 & 1.3487 & $\left(1.6 \times 10^{-6}\right)$ & -0.334 & $\left(1.4 \times 10^{-4}\right)$ & 0.0006 & $\left(5.6 \times 10^{-2}\right)$ \\
\hline 0.2 & 2.1814 & 1.30194 & 0.0027 & 1.3022 & $\left(1.2 \times 10^{-6}\right)$ & -0.275 & $\left(1.5 \times 10^{-4}\right)$ & 0.0027 & $\left(1.7 \times 10^{-2}\right)$ \\
\hline 0.3 & 3.0551 & 1.21559 & 0.0092 & 1.2158 & $\left(3.8 \times 10^{-7}\right)$ & -0.150 & $\left(8.4 \times 10^{-5}\right)$ & 0.0093 & $\left(5.6 \times 10^{-3}\right)$ \\
\hline 0.4 & 3.8129 & 1.13784 & 0.0171 & 1.1381 & $\left(1.5 \times 10^{-6}\right)$ & -0.017 & $\left(1.0 \times 10^{-2}\right)$ & 0.0172 & $\left(3.4 \times 10^{-3}\right)$ \\
\hline 0.5 & 4.4735 & 1.06797 & 0.0252 & 1.0682 & $\left(3.3 \times 10^{-6}\right)$ & 0.123 & $\left(5.1 \times 10^{-3}\right)$ & 0.0254 & $\left(2.7 \times 10^{-3}\right)$ \\
\hline 0.6 & 5.0526 & 1.00519 & 0.0329 & 1.0055 & $\left(2.6 \times 10^{-6}\right)$ & 0.277 & $\left(4.6 \times 10^{-3}\right)$ & 0.0331 & $\left(2.7 \times 10^{-3}\right)$ \\
\hline 0.7 & 5.5643 & 0.94885 & 0.0401 & 0.9488 & $\left(1.3 \times 10^{-5}\right)$ & 0.444 & $\left(1.4 \times 10^{-2}\right)$ & 0.0400 & $\left(2.2 \times 10^{-3}\right)$ \\
\hline 0.8 & 6.0177 & 0.89789 & 0.0467 & 0.8978 & $\left(1.1 \times 10^{-5}\right)$ & 0.604 & $\left(9.9 \times 10^{-3}\right)$ & 0.0465 & $\left(1.9 \times 10^{-3}\right)$ \\
\hline 0.9 & 6.4222 & 0.85177 & 0.0526 & 0.8517 & $\left(9.4 \times 10^{-6}\right)$ & 0.767 & $\left(7.4 \times 10^{-3}\right)$ & 0.0525 & $\left(1.5 \times 10^{-3}\right)$ \\
\hline 1 & 6.7851 & 0.80991 & 0.0580 & 0.8098 & $\left(7.5 \times 10^{-6}\right)$ & 0.932 & $\left(5.6 \times 10^{-3}\right)$ & 0.0581 & $\left(2.0 \times 10^{-4}\right)$ \\
\hline 1.5 & 8.1490 & 0.64847 & 0 & 0.6484 & $\left(5.4 \times 10^{-6}\right)$ & 1.7 & $\left(6.5 \times 10^{-4}\right)$ & 0.0790 & $\left(5.3 \times 10^{-4}\right)$ \\
\hline 2 & 9.0407 & 0.53958 & 0 & 0.5395 & $\left(2.1 \times 10^{-5}\right)$ & 2.64 & $\left(5.7 \times 10^{-3}\right)$ & 0.0920 & $\left(1.1 \times 10^{-3}\right)$ \\
\hline 3 & 10.1299 & 0.40306 & 0 & 0.4030 & $\left(1.7 \times 10^{-5}\right)$ & 4.27 & $\left(1.6 \times 10^{-2}\right)$ & 0.1085 & $\left(1.7 \times 10^{-4}\right)$ \\
\hline 4 & 10.7675 & & & 0.3212 & $\left(1.4 \times 10^{-5}\right)$ & 6.85 & $\left(1.2 \times 10^{-2}\right)$ & 0.1182 & $\left(7.9 \times 10^{-4}\right)$ \\
\hline 5 & 11.1856 & 0.26704 & 0.1238 & 0.2668 & $\left(2.9 \times 10^{-7}\right)$ & 8.44 & $\left(1.0 \times 10^{-3}\right)$ & 0.1220 & $\left(2.1 \times 10^{-3}\right)$ \\
\hline 6 & 11.4800 & 0.22839 & 0.1280 & 0.2281 & $\left(1.4 \times 10^{-6}\right)$ & 10.3 & $\left(1.2 \times 10^{-4}\right)$ & 0.1261 & $\left(1.8 \times 10^{-3}\right)$ \\
\hline 7 & 11.6985 & 0.19949 & 0.1311 & 0.1992 & $\left(3.3 \times 10^{-6}\right)$ & 12.2 & $\left(7.2 \times 10^{-4}\right)$ & 0.1290 & $\left(1.5 \times 10^{-3}\right)$ \\
\hline 8 & 11.8671 & 0.17707 & 0.1334 & 0.1767 & $\left(5.4 \times 10^{-6}\right)$ & 14.1 & $\left(1.5 \times 10^{-3}\right)$ & 0.1312 & $\left(1.3 \times 10^{-3}\right)$ \\
\hline 9 & 12.0011 & 0.15917 & 0.1352 & 0.1588 & $\left(7.7 \times 10^{-6}\right)$ & 16.0 & $\left(2.2 \times 10^{-3}\right)$ & 0.1328 & $\left(1.1 \times 10^{-3}\right)$ \\
\hline 10 & 12.1101 & 0.14455 & 0.1367 & 0.1442 & $\left(1.0 \times 10^{-5}\right)$ & 17.8 & $\left(3.0 \times 10^{-3}\right)$ & 0.1341 & $\left(8.7 \times 10^{-4}\right)$ \\
\hline
\end{tabular}

Since we have solved the leading-order inner problem numerically, it is possible to obtain $c_{i}$ $(i=1,2,3)$ directly from the numerical solution by using Eq. (31). Let us consider the following $\Gamma_{i}$ $(i=1,2,3)$ :

$$
\begin{aligned}
& \Gamma_{1}(r)=\frac{1}{2}\left(\tilde{u}_{r(1)}-2 \tilde{u}_{t(1)}-3\right), \\
& \Gamma_{2}(r)=\frac{1}{2}\left(\tilde{u}_{r(1)}+2 \tilde{u}_{t(1)}+1\right), \\
& \Gamma_{3}(r)=\tilde{\tau}_{(1)} .
\end{aligned}
$$

As seen from Eq. (31), the graph of $y=\ln \left|\Gamma_{i}\right|$ as a function of $x=\ln r$ should approach, with the increase of $r$, a straight line with slope $\alpha_{i}$, where $\alpha_{1}=-1, \alpha_{2}=-3$, and $\alpha_{3}=-2$. The intersection of this straight line with the axis $x=0$ should be $\ln \left|c_{i}\right|$. Therefore, if we plot numerically obtained $\Gamma_{i}(r)$ in a double-log plot and draw a straight line that fits to the plot in a certain interval at large $r$, the slope and the $y$ intercept of the fitting line give the power $\alpha_{i}$ and the (absolute) value of $c_{i}$, respectively. The sign of $c_{i}$ can be identified from the sign of $\Gamma_{i}$. As for the fitting method, the least-square method was used.

The values of $c_{i}(i=1,2,3)$ thus obtained are shown in Table III for $\operatorname{Pr}=2 / 3$ and in Table IV for $\operatorname{Pr}=1$ (see the columns "direct computation"). As seen from the tables, the overall agreement between the values of $c_{1}$ and $c_{3}$ obtained by the direct method and those obtained by the formulas (40) 
TABLE IV. $h_{D}, c_{1}, c_{2}$, and $c_{3}$ vs $k$ for the BGK model (or the ES model with $\operatorname{Pr}=1$ ) under the diffuse reflection boundary condition. See the caption of Table III.

\begin{tabular}{|c|c|c|c|c|c|c|c|c|c|}
\hline \multirow[b]{2}{*}{$k$} & \multirow[b]{2}{*}{$h_{D}$} & \multirow{2}{*}{$\begin{array}{l}\text { Formula (40) } \\
\qquad-c_{1}\end{array}$} & \multirow{2}{*}{$\begin{array}{c}\text { Formula (42) } \\
\quad-c_{3}\end{array}$} & \multicolumn{6}{|c|}{ Direct computation } \\
\hline & & & & & $-c_{1}$ & & $-c_{2}$ & & $-c_{3}$ \\
\hline 0.1 & 1.7056 & 1.35724 & -0.0032 & 1.3572 & $\left(6.0 \times 10^{-7}\right)$ & -0.349 & $\left(8.7 \times 10^{-5}\right)$ & -0.0032 & $\left(5.9 \times 10^{-4}\right)$ \\
\hline 0.15 & 2.4378 & 1.29328 & -0.0 & 1.2935 & $\left(3.7 \times 10^{-6}\right)$ & -0.274 & $\left(6.4 \times 10^{-4}\right)$ & -0.0030 & $\left(5.3 \times 10^{-3}\right)$ \\
\hline 0.2 & 3.1008 & 1.23375 & -0.0 & 39 & $\left(1.5 \times 10^{-6}\right)$ & -0.197 & $\left(1.0 \times 10^{-3}\right)$ & -0.0007 & $\left(4.5 \times 10^{-2}\right)$ \\
\hline 0.3 & 4.2446 & 1.1 & 9 & 1.1 & $\left(1.8 \times 10^{-7}\right)$ & -0.036 & $\left(1.8 \times 10^{-3}\right)$ & 0.0079 & $\left(9.0 \times 10^{-3}\right)$ \\
\hline 0.4 & 5.1864 & 1.03179 & 0.0185 & 1.0320 & $\left(4.0 \times 10^{-7}\right)$ & 0.131 & $\left(5.9 \times 10^{-4}\right)$ & 0.0186 & $\left(1.9 \times 10^{-3}\right)$ \\
\hline 0.5 & 5.9679 & 0.94982 & 0.0292 & 0.9501 & $\left(8.0 \times 10^{-7}\right)$ & 0.305 & $\left(6.6 \times 10^{-4}\right)$ & 0.0295 & $\left(2.2 \times 10^{-3}\right)$ \\
\hline 0.6 & 6.6228 & 0.87838 & 0.0391 & 0.8787 & $\left(2.6 \times 10^{-6}\right)$ & 0.483 & $\left(1.6 \times 10^{-3}\right)$ & 0.0394 & $\left(3.0 \times 10^{-3}\right)$ \\
\hline 0.7 & 7.1781 & 0.81602 & 0.0480 & 0.8160 & $\left(2.6 \times 10^{-6}\right)$ & 0.672 & $\left(1.9 \times 10^{-3}\right)$ & 0.0479 & $\left(1.4 \times 10^{-3}\right)$ \\
\hline 0.8 & 7.6522 & 0.76117 & 0.0559 & 0.7611 & $\left(6.9 \times 10^{-7}\right)$ & 0.856 & $\left(9.3 \times 10^{-4}\right)$ & 0.0560 & $\left(4.6 \times 10^{-5}\right)$ \\
\hline 0.9 & 8.0611 & 0.71275 & 0.0629 & 0.7127 & $\left(1.4 \times 10^{-6}\right)$ & 1.04 & $\left(1.2 \times 10^{-4}\right)$ & 0.0632 & $\left(1.3 \times 10^{-3}\right)$ \\
\hline 1 & 8.4168 & 0.66979 & 0.0691 & 0.6697 & $\left(4.0 \times 10^{-6}\right)$ & 1.23 & $\left(7.5 \times 10^{-4}\right)$ & 0.0691 & $\left(1.0 \times 10^{-3}\right)$ \\
\hline 1.5 & 9.6637 & 0.51268 & 0.0913 & 0.5126 & $\left(1.1 \times 10^{-5}\right)$ & 2.16 & $\left(4.2 \times 10^{-3}\right)$ & 0.0917 & $\left(1.0 \times 10^{-3}\right)$ \\
\hline 2 & 10.4056 & 0.41403 & 0.1046 & 0.4140 & $\left(8.7 \times 10^{-6}\right)$ & 3.25 & $\left(7.0 \times 10^{-3}\right)$ & 0.1043 & $\left(1.2 \times 10^{-3}\right)$ \\
\hline 3 & 11.2381 & 0.29810 & 0.1193 & 0.2980 & $\left(5.2 \times 10^{-6}\right)$ & 5.20 & $\left(3.9 \times 10^{-3}\right)$ & 0.1193 & $\left(1.6 \times 10^{-4}\right)$ \\
\hline 4 & 11.6897 & 0.23256 & 0.1271 & 0.2325 & $\left(2.8 \times 10^{-6}\right)$ & 7.14 & $\left(2.3 \times 10^{-3}\right)$ & 0.1275 & $\left(8.5 \times 10^{-4}\right)$ \\
\hline 5 & 11.9721 & 0.19054 & 0.1319 & 0.1904 & $\left(6.7 \times 10^{-6}\right)$ & 8.91 & $\left(2.4 \times 10^{-3}\right)$ & 0.1298 & $\left(2.3 \times 10^{-3}\right)$ \\
\hline 6 & 12.1645 & 0.16134 & 0.1351 & 0.1611 & $\left(8.8 \times 10^{-6}\right)$ & 10.8 & $\left(2.9 \times 10^{-3}\right)$ & 0.1329 & $\left(2.0 \times 10^{-3}\right)$ \\
\hline 7 & 12.3040 & 0.13987 & 0.1374 & 0.1396 & $\left(1.1 \times 10^{-5}\right)$ & 12.7 & $\left(3.4 \times 10^{-3}\right)$ & 0.1351 & $\left(1.7 \times 10^{-3}\right)$ \\
\hline 8 & 12.4096 & 0.12344 & 0.13 & 0.1232 & $\left(1.0 \times 10^{-5}\right)$ & 14.6 & $\left(3.9 \times 10^{-3}\right)$ & 0.1366 & $\left(1.5 \times 10^{-3}\right)$ \\
\hline 9 & 12.4923 & 0.11046 & 01404 & 0.1102 & $\left(9.2 \times 10^{-6}\right)$ & 16.4 & $\left(4.4 \times 10^{-3}\right)$ & 0.1378 & $\left(1.2 \times 10^{-3}\right)$ \\
\hline 10 & 12.5588 & 0.09994 & 0.1415 & 0.0996 & $\left(8.5 \times 10^{-6}\right)$ & 18.3 & $\left(5.0 \times 10^{-3}\right)$ & 0.1386 & $\left(9.9 \times 10^{-4}\right)$ \\
\hline
\end{tabular}

and (42) is satisfactory. The larger discrepancy in the values for larger $k$ is attributed to the difficulty of the numerical computation in this regime, due to the need of increasingly wider computational domain for $r$ variable. As for $c_{2}$, the sign is not definite but is changed at a relatively small value of $k$. The magnitude of $c_{2}$ seems to increase indefinitely. However, it should be noted that it is much harder to obtain $c_{2}$ in a numerical computation, because the magnitude of $\Gamma_{2}$ is much smaller than that of $\Gamma_{1}$ or $\Gamma_{3}$ when $r$ is large, due to its faster decay $\left(\sim r^{-3}\right)$. Thus, the definite conclusion for the trend of $c_{2}$ requires a further investigation. Finally, we remark that the deviation of the slope of the fitting line from $\alpha_{i}$ can be viewed as a measure of accuracy of the present computations. They are shown in Tables III and IV in the parentheses.

We have so far presented $h_{D}$ and $c_{i}(i=1,2,3)$ for the ES model with different Prandtl numbers $\left(\operatorname{Pr}=2 / 3\right.$ in Table III and $\operatorname{Pr}=1$ in Table IV). We also showed $h_{D}, c_{1}$, and $c_{3}$ for the hard-sphere gas (Table II). A way of comparing these results is to regard the viscosity or the thermal conductivity as the common basic quantity. Noting that the viscosity (or the thermal conductivity) is proportional to $\gamma_{1} \ell_{\infty}\left(\right.$ or $\gamma_{2} \ell_{\infty}$ ) at the same reference state [see Eqs. (A7) in Appendix A], the Knudsen number $k$ for the hard-sphere gas, that for the ES model, and that for the BGK model (or the ES model with $\operatorname{Pr}=1$ ) can be related each other by either of the relations

$$
\gamma_{1}^{\mathrm{HS}} k^{\mathrm{HS}}=\operatorname{Pr} k^{\mathrm{ES}}=k^{\mathrm{BGK}}, \quad \gamma_{2}^{\mathrm{HS}} k^{\mathrm{HS}}=k^{\mathrm{ES}}=k^{\mathrm{BGK}},
$$

where $\gamma_{1}^{\mathrm{HS}}=1.270042427$ and $\gamma_{2}^{\mathrm{HS}}=1.922284066$ (see Appendix A). With either of them, we convert $k$ for the ES or BGK model to that for the hard-sphere model, thereby recasting $h_{D}(k)$, $c_{1}(k), c_{2}(k)$, and $c_{3}(k)$ for the ES or BGK model as functions of $k=k^{\mathrm{HS}}$. Functions $h_{D}(k)$ and $c_{i}(k)$ for the ES or BGK model thus obtained are shown in Fig. $2\left(h_{D}\right.$ and $\left.c_{1}\right)$ and in Fig. $3\left(c_{2}\right.$ and $\left.c_{3}\right)$, together with $\left(h_{D}, c_{1}, c_{3}\right)$ for the hard-sphere model in Fig. 2 and in Fig. 3(b). Note that 


\section{SATOSHI TAGUCHI AND TOSHIHIRO SUZUKI}

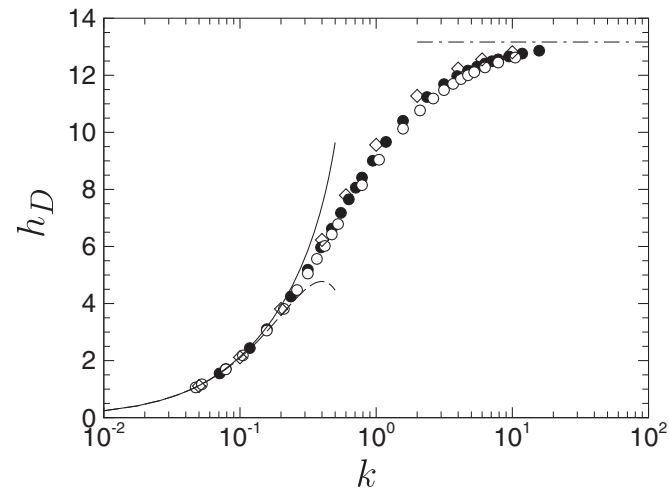

(a)

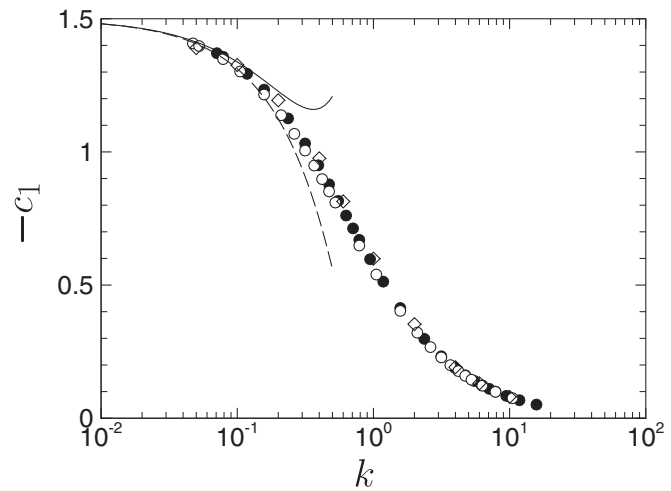

(b)

FIG. 2. $h_{D}(k)$ and $c_{1}(k)$ under the diffuse reflection condition for the hard-sphere gas $(\diamond)$, for the ES model with $\operatorname{Pr}=2 / 3(\circ)$, and for the ES model with $\operatorname{Pr}=1$ (or the BGK model) $\left(\bullet\right.$ ). The value of $h_{D}$ for the hard-sphere gas were taken from Ref. [22] (also shown in Table II). The solid line indicates the asymptotic formulas (34) and (35) for the hard-sphere gas. The dashed line shows the first two terms of the formulas. In (a), the asymptotic value as $k \rightarrow \infty$ is also indicated by dash-dotted line.

the viscosity-based conversion was used for $h_{D}, c_{1}$, and $c_{2}$, while the thermal-conductivity-based conversion for $c_{3}$. As seen from Fig. 2, the difference between the models is rather small for $h_{D}$ and $c_{1}$ [38]. In Fig. 3 a similar comparison for the functions $c_{2}(k)$ and $c_{3}(k)$ are made. The difference between the models is more pronounced in $c_{2}$ and in $c_{3}$ than in $h_{D}$ and in $c_{1}$. In $c_{3}$, the BGK model gives slightly closer results to the hard-sphere model, except for small $k$. Recalling that the function $c_{3}(k)$ is essentially the thermal force $h_{T}$ in the problem of thermophoresis of a sphere, the present result implies sensitivity on the choice of the model parameter Pr, when the ES model is used to analyze this problem.

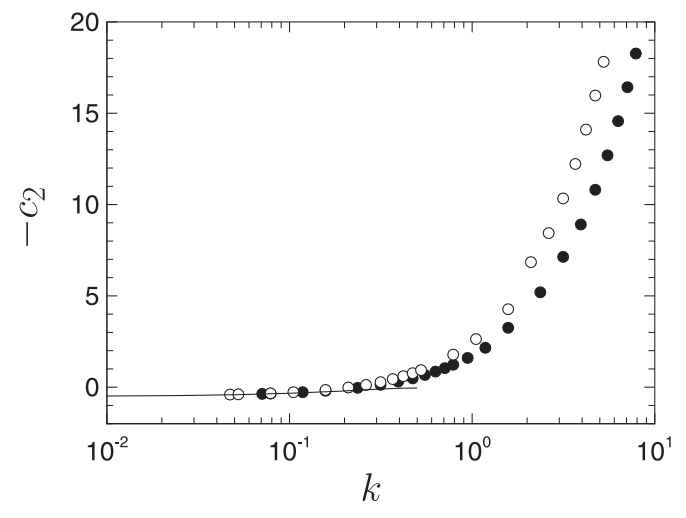

(a)

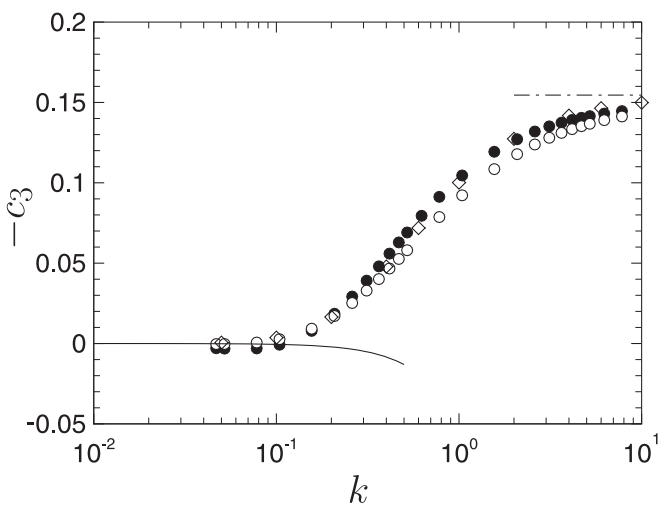

(b)

FIG. 3. $c_{2}(k)$ and $c_{3}(k)$ under the diffuse reflection condition for the ES model with $\operatorname{Pr}=2 / 3(0)$ and for the ES model with $\operatorname{Pr}=1$ (or the BGK model) $(\bullet)$, and $c_{3}(k)$ under the diffuse reflection condition for the hard-sphere gas $(\diamond)$. The solid line indicates the asymptotic formulas (36) and (37) for the hard-sphere gas. In (b), the dash-dotted line indicates the asymptotic value as $k \rightarrow \infty$ for the hard-sphere model. 


\section{CONCLUSION}

In this paper, we have considered a steady rarefied gas flow past a sphere on the basis of kinetic theory, in the case where the velocity of the uniform flow is small (or the Mach number is small). In the preceding paper, the problem was investigated by means of an asymptotic analysis for small Mach numbers, and the expression of the drag up to the second order of the Mach number was derived; the result appears in Eq. (32). In this expression, the effect of gas rarefaction is expressed by the two functions $h_{D}(k)$ and $c_{1}(k)$, the former of which is known from the existing literature for some molecular models. In this paper, we aimed at obtaining $c_{1}(k)$ for the hard-sphere gas as well as for the ES model of the Boltzmann equation under the diffuse reflection boundary condition, thereby completing the second-order drag for these models.

The difficulty in obtaining $c_{1}$ comes from the fact that it is a factor of a decaying term in the solution (the flow velocity), which vanishes at infinity. The same is true for the factor $c_{3}$ describing the temperature in the far field. Therefore, we first derived simple representations of $c_{1}$ and $c_{3}$ in terms of $h_{D}$ (drag) and $h_{T}$ (thermal force), respectively, both of which are known for the hard-sphere gas in the existing literature. We also obtained a representation formula of $c_{3}$ in terms of the quantities on the boundary, which are easier to handle numerically. As the result, we obtained the numerical values of $c_{1}$ and $c_{3}$ as functions of $k$, which are summarized in Tables II-IV. Finally, we remark that the present result for $c_{3}$ can be used to derive new data of $h_{T}$ in the problem of thermophoresis for the ES model with $\operatorname{Pr}=2 / 3$ (under the diffuse reflection condition), due to the relation (44).

\section{ACKNOWLEDGMENTS}

This work was supported by JSPS KAKENHI Grant No. 25820041. S.T also acknowledges the support of the bilateral program (SAKURA Program) between JSPS and MAEDI for his stay in Paris and wishes to thank Prof. Francesco Salvarani and Prof. François Golse for valuable discussions and kind hospitalities. The relation (44) was first verified using the data of $h_{T}$ for the BGK model. We thank Prof. Shigeru Takata for his courtesy of providing us with detailed numerical data on $h_{T}$ for this model.

\section{APPENDIX A: COLLISION INTEGRAL, RELATED FUNCTIONS, AND TRANSPORT COEFFICIENTS}

The linear and nonlinear parts of the collision integral, $\mathcal{L}$ and $\mathcal{J}$, are written in the dimensionless form as [22]

$$
\begin{gathered}
\mathcal{L}[\phi]=\int E_{*}\left(\phi^{\prime}+\phi_{*}^{\prime}-\phi-\phi_{*}\right) B d \Omega(\mathbf{e}) d \zeta_{*}, \\
\mathcal{J}[\phi, \psi]=\frac{1}{2} \int E_{*}\left(\phi^{\prime} \psi_{*}^{\prime}+\phi_{*}^{\prime} \psi^{\prime}-\phi \psi_{*}-\phi_{*} \psi\right) B d \Omega(\mathbf{e}) d \zeta_{*} \\
\phi=\phi\left(\zeta_{i}\right), \quad \phi_{*}=\phi\left(\zeta_{i *}\right), \quad \phi^{\prime}=\phi\left(\zeta_{i}^{\prime}\right), \quad \phi_{*}^{\prime}=\phi\left(\zeta_{i *}^{\prime}\right) \\
\zeta_{i}^{\prime}=\zeta_{i}+e_{i} e_{j}\left(\zeta_{j *}-\zeta_{j}\right), \quad \zeta_{i *}^{\prime}=\zeta_{i *}-e_{i} e_{j}\left(\zeta_{j *}-\zeta_{j}\right)
\end{gathered}
$$

where $E_{*}=\pi^{-3 / 2} \exp \left(-\zeta_{j *}^{2}\right), e_{i}$ (or $\left.\mathbf{e}\right)$ is the unit vector, $d \Omega(\mathbf{e})$ is the solid-angle element in the direction of $e_{i}, d \zeta_{*}=d \zeta_{1 *} d \zeta_{2 *} d \zeta_{3 *}$, and the integral with respect to $\zeta_{i *}$ and $e_{i}$ is carried out over the whole space of $\zeta_{i *}$ and all directions of $e_{i}$. In Eqs. (A1) and (A2),

$$
B=B\left(\left|e_{i}\left(\zeta_{i *}-\zeta_{i}\right)\right| /\left|\zeta_{j *}-\zeta_{j}\right|,\left|\zeta_{j *}-\zeta_{j}\right|\right)
$$

with $\left|\zeta_{j *}-\zeta_{j}\right|=\left[\left(\zeta_{j *}-\zeta_{j}\right)^{2}\right]^{1 / 2}$ is a nonnegative function whose functional form is determined by the intermolecular force. For the hard-sphere gas, $B=\left|e_{i}\left(\zeta_{i *}-\zeta_{i}\right)\right| / 4(2 \pi)^{1 / 2}$. It holds that $\mathcal{L}[\phi]=2 \mathcal{J}[1, \phi]$. 


\section{SATOSHI TAGUCHI AND TOSHIHIRO SUZUKI}

Now let us introduce functions $A(\zeta), B(\zeta), D_{1}(\zeta), D_{2}(\zeta), F(\zeta)$ with $\zeta=\left(\zeta_{j}^{2}\right)^{1 / 2}$ as the solutions of the following integral equations:

$$
\begin{aligned}
& \mathcal{L}\left[\zeta_{i} A\right]=-\zeta_{i}\left(\zeta^{2}-\frac{5}{2}\right), \quad \text { with }\left\langle\zeta^{2} A\right\rangle=0, \\
& \mathcal{L}\left[\zeta_{i j} B\right]=-2 \zeta_{i j}, \\
& \mathcal{L}\left[\zeta_{i j} F\right]=\zeta_{i j} A, \\
& \mathcal{L}\left[\left(\zeta_{i} \delta_{j k}+\zeta_{j} \delta_{k i}+\zeta_{k} \delta_{i j}\right) D_{1}+\zeta_{i} \zeta_{j} \zeta_{k} D_{2}\right] \\
& \quad=\gamma_{1}\left(\zeta_{i} \delta_{j k}+\zeta_{j} \delta_{k i}+\zeta_{k} \delta_{i j}\right)-\zeta_{i} \zeta_{j} \zeta_{k} B, \quad \text { with }\left\langle\zeta^{2}\left(5 D_{1}+\zeta^{2} D_{2}\right)\right\rangle=0,
\end{aligned}
$$

where $\zeta_{i j}=\zeta_{i} \zeta_{j}-\left(\zeta^{2} / 3\right) \delta_{i j}$, and $\gamma_{1}$ in Eq. (A5d) is given subsequently. Then, $\gamma_{i}(i=1,2,3)$ are defined as

$$
\begin{aligned}
& \gamma_{1}=\frac{2}{15}\left\langle\zeta^{4} B\right\rangle, \quad \gamma_{2}=\frac{4}{15}\left\langle\zeta^{4} A\right\rangle \\
& \gamma_{3}=\frac{2}{15}\left\langle\zeta^{4} A B\right\rangle=\frac{2}{3}\left\langle\zeta^{4} D_{1}\right\rangle+\frac{2}{15}\left\langle\zeta^{6} D_{2}\right\rangle=-\frac{4}{15}\left\langle\zeta^{4} F\right\rangle .
\end{aligned}
$$

The $\gamma_{1}$ and $\gamma_{2}$ correspond physically to the viscosity and thermal conductivity of the gas. That is, the viscosity $\mu_{*}$ and the thermal conductivity $\lambda_{*}$ at the reference state (with temperature $T_{*}$ and pressure $\left.p_{*}\right)$ are given by

$$
\begin{aligned}
& \mu_{*}=(\sqrt{\pi} / 2) \gamma_{1} p_{*}\left(2 R T_{*}\right)^{-1 / 2} \ell_{*}, \\
& \lambda_{*}=(5 \sqrt{\pi} / 4) \gamma_{2} p_{*}\left(2 R T_{*}\right)^{-1 / 2} R \ell_{*},
\end{aligned}
$$

where $\ell_{*}$ is the mean free path of gas molecules at the reference equilibrium state at rest. The $\gamma$ values for the hard-sphere gas are [22]

$$
\gamma_{1}=1.270042427, \quad \gamma_{2}=1.922284066, \quad \gamma_{3}=1.947906335 \text {. }
$$

The Prandtl number Pr is defined by

$$
\operatorname{Pr}=\frac{5 R}{2} \frac{\mu_{*}}{\lambda_{*}}=\frac{\gamma_{1}}{\gamma_{2}}
$$

and therefore $\operatorname{Pr}=0.660694457$ for the hard-sphere model.

The linearized collision integral $\mathcal{L}^{\mathrm{ES}}$ for the ES model is written as

$$
\mathcal{L}^{\mathrm{ES}}[\phi]=-\phi+\langle\phi\rangle+2\left\langle\zeta_{i} \phi\right\rangle \zeta_{i}+\frac{2}{3}\left\langle\left(\zeta^{2}-\frac{3}{2}\right) \phi\right\rangle\left(\zeta^{2}-\frac{3}{2}\right)+2 v\left\langle\zeta_{i j} \phi\right\rangle \zeta_{i} \zeta_{j},
$$

where $v \in[-1 / 2,1)$ is a parameter. The explicit form of $\mathcal{J}$ for this model is omitted because it is not required in this paper. The functions $A, B, D_{1}, D_{2}, F$ as well as the $\gamma$ for the ES model are easily obtained [24] and are summarized as follows:

$$
\begin{aligned}
A & =\zeta^{2}-\frac{5}{2}, \quad B=\frac{2}{1-v}=2 \operatorname{Pr}, \quad D_{1}=-\frac{1}{1-v}=-\operatorname{Pr}, \quad D_{2}=\frac{2}{1-v}=2 \operatorname{Pr} \\
F & =-\zeta^{2}+\frac{5}{2}-\frac{v}{1-v}=-\zeta^{2}+\frac{7}{2}-\operatorname{Pr} \\
\gamma_{1} & =\frac{1}{1-v}=\operatorname{Pr}, \quad \gamma_{2}=1, \quad \gamma_{3}=\frac{1}{1-v}=\operatorname{Pr} .
\end{aligned}
$$


The Prandtl number for the ES model is given by

$$
\operatorname{Pr}=\frac{\gamma_{1}}{\gamma_{2}}=\frac{1}{1-v}
$$

This relation was used in Eq. (A10).

The BGK model of the collision integral is obtained as a special case of the ES model by setting $v=0$ (or $\operatorname{Pr}=1$ ). Therefore, the corresponding results for the BGK model are readily obtained by setting $v=0$ (or $\operatorname{Pr}=1$ ) in Eq. (A10).

\section{APPENDIX B: NUMERICAL ANALYSIS}

For the purpose of numerical analysis, it is convenient to introduce the polar angle $\theta_{\zeta}=$ $\cos ^{-1}\left(\zeta_{r} / \zeta\right)\left(0 \leqslant \theta_{\zeta} \leqslant \pi\right)$ for the molecular velocity and regard $\Phi=\left(\varphi_{1 a}, \varphi_{1 b}\right)$ as a function of $\left(r, \theta_{\zeta}, \zeta\right)$. Also for the same purpose, we introduce upper limits for the variables $r$ and $\zeta$, and consider the solution in the finite domain $\left(r, \theta_{\zeta}, \zeta\right) \in\left[1, r_{D}\right] \times[0, \pi] \times\left[0, \zeta_{D}\right]$, where $r_{D}$ and $\zeta_{D}$ are appropriately chosen positive constants whose validity should be verified from the numerical result.

One of important aspects in the present problem is that VDF is discontinuous in the gas, which is a feature commonly observed in a gas around a convex body [39]. Recently, it has been shown that such a discontinuity in VDF over a smooth convex boundary is responsible for the singular behavior of the macroscopic quantities on the boundary [40]. More precisely, the normal derivative of the macroscopic quantities diverges on a convex boundary. Therefore, appropriate care to the discontinuity in VDF in a numerical analysis is not only of theoretical interest, but also is important for the accurate evaluation of the macroscopic quantities on the boundary. The finite-difference method developed in Ref. [11] and used in the present computation, which is also based on similar methods in Refs. [41-44], has an ability to capture such discontinuities in VDF and therefore is suitable for the purpose of the present study.

In the present problem, $\lim _{\theta_{\zeta} \rightarrow \pi / 2+0} \Phi \neq \lim _{\theta_{\zeta} \rightarrow \pi / 2-0} \Phi$ at $r=1$ (i.e., on the sphere) for any fixed $\zeta>0$, and this discontinuity propagates in the $\left(r, \theta_{\zeta}\right)$ plane along the curve $r \sin \theta_{\zeta}=1$ $\left(0<\theta_{\zeta}<\pi / 2\right)$. With this observation, we divide the domain of computation into three regions I, II, and III defined by

$$
\begin{aligned}
& \text { I }:\left\{\left(r, \theta_{\zeta}\right): 1 \leqslant r \leqslant r_{D}, 0<\theta_{\zeta} \leqslant \pi / 2, r \sin \theta_{\zeta}<1\right\}, \\
& \text { II }:\left\{\left(r, \theta_{\zeta}\right): 1 \leqslant r \leqslant r_{D}, 0<\theta_{\zeta} \leqslant \pi / 2, r \sin \theta_{\zeta}>1\right\}, \\
& \text { III }:\left\{\left(r, \theta_{\zeta}\right): 1 \leqslant r \leqslant r_{D}, \pi / 2<\theta_{\zeta} \leqslant \pi\right\}
\end{aligned}
$$

(see Fig. 4). Here, the domains I and II are separated by the curve of discontinuity $r \sin \theta_{\zeta}=1$, and the domains II and III are separated by the line $\theta_{\zeta}=\pi / 2$. By so doing, there is no discontinuity in the interior of the subregions. Therefore, we may apply the usual finite-difference scheme on a rectangular grid in each region without crossing the curve of discontinuity. On the other hand, as the result of the domain decomposition, we require, as the boundary condition, the limiting values of $\Phi$ on both sides of the curve $r \sin \theta_{\zeta}=1$, where VDF becomes discontinuous. Let us denote these limiting values by $\Phi^{ \pm}=\left.\Phi\right|_{\theta_{\zeta} \rightarrow \sin ^{-1}(1 / r) \pm 0}$. Then, $\Phi^{ \pm}$is obtained by integrating the equation along the characteristic $r \sin \theta_{\zeta}=1\left(0<\theta_{\zeta}<\pi / 2\right)$ starting from $r=1$, with the initial value $\left.\Phi^{ \pm}\right|_{r=1}$.

In the last process, it is advantageous to compute only $\Phi^{+}$numerically and then to obtain $\Phi^{-}$by the formula $\Phi^{-}=\Phi^{+}-\left(\Phi^{+}-\Phi^{-}\right)=\Phi^{+}-[\Phi]$, where $[\Phi]=\Phi^{+}-\Phi^{-}$is the amount of jump. The $[\Phi]$ is explicitly given by

$$
\begin{aligned}
& {\left[\varphi_{1 a}\right]= \begin{cases}\left(\left[\varphi_{1 a}\right]_{0} r \sin ^{2} \theta_{\zeta}-\left[\varphi_{1 b}\right]_{0} \zeta \cos \theta_{\zeta}\right) \exp \left(-\frac{s}{k \zeta}\right), & (\zeta>0), \\
0, & (\zeta=0),\end{cases} } \\
& {\left[\varphi_{1 b}\right]= \begin{cases}\left(\zeta^{-1}\left[\varphi_{1 a}\right]_{0} r \cos \theta_{\zeta}+\left[\varphi_{1 b}\right]_{0}\right) \exp \left(-\frac{s}{k \zeta}\right), & (\zeta>0), \\
0, & (\zeta=0),\end{cases} }
\end{aligned}
$$




\section{SATOSHI TAGUCHI AND TOSHIHIRO SUZUKI}

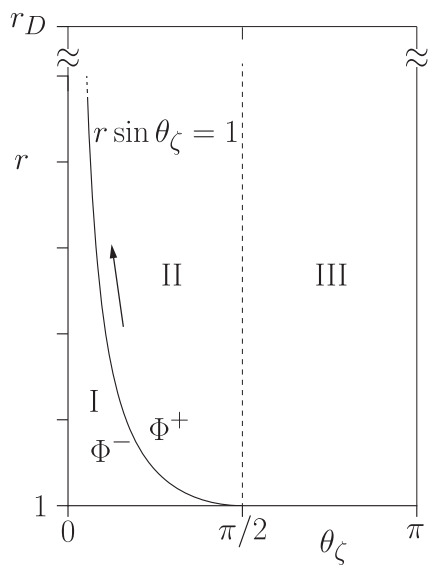

FIG. 4. The computational domain. The domains II and III are separated by the characteristic $r \sin \theta_{\zeta}=1$ $\left(0<\theta_{\zeta} \leqslant \pi / 2\right)$. The arrow indicates the direction of propagation of the discontinuity in VDF.

$$
\begin{aligned}
{\left[\varphi_{1 \alpha}\right]_{0} } & =\varphi_{1 \alpha}\left(r=1, \theta_{\zeta}=\pi / 2+0, \zeta\right)-\varphi_{1 \alpha}\left(r=1, \theta_{\zeta}=\pi / 2-0, \zeta\right), \quad(\alpha=a, b), \\
s & =r \cos \theta_{\zeta}
\end{aligned}
$$

This is because when $\zeta(>0)$ is small, $\Phi^{-}$varies abruptly near $r=1$ (the equation becomes stiff), and therefore many grid points are required. Incidentally, Eq. (B2) is derived by integrating the equation for the jump along $r=1 / \sin \theta_{\zeta}$ from $r=1$ to $r$, assuming that the gain terms of the equations for $\Phi^{ \pm}$are continuous (and thus do not contribute to the equation for the jump). In addition, there are a number of other devises used to help improve the accuracy of the numerical solution. We omit the details because they are essentially the same as those used in Ref. [11]. The total number of grid points used in the computations for the result presented in the paper is summarized as follows: for the domain $\left(r, \theta_{\zeta}, \zeta\right), 1600 \times 512 \times 72$ subdivisions were used for $k<5$ and $3200 \times 512 \times 72$ subdivisions for $k \geqslant 5$. The upper limit of $r$, i.e., $r_{D}$, was set to be 150 times larger than $k$ or greater, depending on $k$. For the upper limit $\zeta_{D}$ for $\zeta, \zeta_{D}=5.4$ was used throughout the computations.

[1] S. Kaplun and P. Lagerstrom, Asymptotic expansions of Navier-Stokes solutions for small Reynolds numbers, Indiana Univ. Math. J. 6, 585 (1957).

[2] I. Proudman and J. R. A. Pearson, Expansions at small Reynolds numbers for the flow past a sphere and a circular cylinder, J. Fluid Mech. 2, 237 (1957).

[3] W. Chester, D. R. Breach, and I. Proudman, On the flow past a sphere at low Reynolds number, J. Fluid Mech. 37, 751 (1969).

[4] C. Cercignani, C. D. Pagani, and P. Bassanini, Flow of a rarefied gas past an axisymmetric body. II. Case of a sphere, Phys. Fluids 11, 1399 (1968).

[5] Y. Sone and K. Aoki, Forces on a spherical particle in a slightly rarefied gas, in Rarefied Gas Dynamics, Progress in Astronautics and Aeronautics, edited by J. L. Potter (AIAA, New York, 1977), pp. 417-433.

[6] Y. Sone and K. Aoki, Slightly rarefied gas flow over a specularly reflecting body, Phys. Fluids 20, 571 (1977).

[7] W. S. Law and S. K. Loyalka, Motion of a sphere in a rarefied gas. II. Role of temperature variation in the Knudsen layer, Phys. Fluids 29, 3886 (1986).

[8] K. Aoki and Y. Sone, Temperature field induced around a sphere in a uniform flow of a rarefied gas, Phys. Fluids 30, 2286 (1987). 
[9] S. A. Beresnev, V. G. Chernyak, and G. A. Fomyagin, Motion of a spherical particle in a rarefied gas. Part 2. Drag and thermal polarization, J. Fluid Mech. 219, 405 (1990).

[10] S. K. Loyalka, Motion of a sphere in a gas: Numerical solution of the linearized Boltzmann equation, Phys. Fluids A 4, 1049 (1992).

[11] S. Takata, Y. Sone, and K. Aoki, Numerical analysis of a uniform flow of a rarefied gas past a sphere on the basis of the Boltzmann equation for hard-sphere molecules, Phys. Fluids A 5, 716 (1993).

[12] M. Torrilhon, Slow gas microflow past a sphere: Analytical solution based on moment equations, Phys. Fluids 22, 072001 (2010).

[13] A. N. Volkov, Transitional flow of a rarefied gas over a spinning sphere, J. Fluid Mech. 683, 320 (2011).

[14] S. Taguchi, Asymptotic theory of a uniform flow of a rarefied gas past a sphere at low Mach numbers, J. Fluid Mech. 774, 363 (2015).

[15] P. L. Bhatnagar, E. P. Gross, and M. Krook, A model for collision processes in gases. I. Small amplitude processes in charged and neutral one-component systems, Phys. Rev. 94, 511 (1954).

[16] P. Welander, On the temperature jump in a rarefied gas, Ark. Fys. 7, 507 (1954).

[17] L. H. Holway, Jr., Approximation procedures for kinetic theory, Ph.D. thesis, Harvard University, 1963.

[18] J. Holway, New statistical models for kinetic theory: Methods of construction, Phys. Fluids 9, 1658 (1966).

[19] D. R. Willis, Sphere drag at high Knudsen number and low Mach number, Phys. Fluids 9, 2522 (1966).

[20] K. C. Lea and S. K. Loyalka, Motion of a sphere in a rarefied gas, Phys. Fluids 25, 1550 (1982).

[21] Y. Sone and K. Aoki, A similarity solution of the linearized Boltzmann equation with application to thermophoresis of a spherical particle, J. Méc. Théor. Appl. 2, 3 (1983).

[22] Y. Sone, Molecular Gas Dynamics: Theory, Techniques, and Applications (Birkhäuser, Boston, 2007); Supplementary Notes and Errata, Kyoto University Research Information Repository, http://hdl.handle.net/2433/66098.

[23] Y. Sone, Kinetic Theory and Fluid Dynamics (Birkhäuser, Boston, 2002), Supplementary Notes and Errata, Kyoto University Research Information Repository, http://hdl.handle.net/2433/66099.

[24] S. Takata, M. Hattori, and T. Hasebe, Slip/jump coefficients and Knudsen-layer corrections for the ES model in the generalized slip-flow theory, AIP Conf. Proc. 1786, 040004 (2016).

[25] S. Takata and M. Hattori, Asymptotic theory for the time-dependent behavior of a slightly rarefied gas over a smooth solid boundary, J. Stat. Phys. 147, 1182 (2012).

[26] S. Takata and M. Hattori, On the second-order slip and jump coefficients for the general theory of slip flow, AIP Conf. Proc. 1501, 59 (2012).

[27] S. Takata and M. Hattori, Numerical data for the generalized slip-flow theory, Kyoto University Research Information Repository, http://hdl.handle.net/2433/199811 (2015).

[28] P. S. Epstein, Zur theorie des radiometers, Z. Phys. 54, 537 (1929).

[29] S. Takata, K. Aoki, and Y. Sone, Thermophoresis of a sphere with a uniform temperature: Numerical analysis of the Boltzmann equation for hard-sphere molecules, in Rarefied Gas Dynamics: Theory and Simulations, edited by Bernie D. Shizgal and David P. Weaver (AIAA, Washington, D.C., 1994), pp. 626-639.

[30] S. Takata, Note on the relation between thermophoresis and slow uniform flow problems for a rarefied gas, Phys. Fluids 21, 112001 (2009).

[31] S. Takata, Symmetry of the linearized Boltzmann equation and its application, J. Stat. Phys. 136, 751 (2009).

[32] C. Cercignani, The Boltzmann Equation and Its Applications (Springer-Verlag, Berlin, 1988).

[33] P. Andries, P. Le Tallec, J.-P. Perlat, and B. Perthame, The Gaussian-BGK model of Boltzmann equation with small Prandtl number, Eur. J. Mech. B/Fluids 19, 813 (2000).

[34] S. Brull and J. Schneider, A new approach for the ellipsoidal statistical model, Continuum Mech. Thermodyn. 20, 63 (2008).

[35] S. Brull, An ellipsoidal statistical model for gas mixtures, Commun. Math. Sci. 13, 1 (2015).

[36] Y. Sone, Flow induced by thermal stress in rarefied gas, Phys. Fluids 15, 1418 (1972).

[37] Y. Sone and K. Aoki, Negative thermophoresis: Thermal stress slip flow around a spherical particle in a rarefied gas, in Rarefied Gas Dynamics, edited by S. S. Fisher (AIAA, New York, 1981), pp. 489-503. 


\section{SATOSHI TAGUCHI AND TOSHIHIRO SUZUKI}

[38] The slip coefficients $b_{i}^{(1)}(i=1,4,5,6)$ and the Knudsen-layer function $Y_{1}^{(1)}$ occurring in the asymptotic expressions of $h_{D}, c_{1}$, and $c_{2}$ for small $k \ll 1$, Eqs. (34)-(36), are self-similar for the ES model in the sense $\left(b_{i}^{(1)}, Y_{1}^{(1)}\right)^{\mathrm{ES}}=\operatorname{Pr}\left(b_{i}^{(1)}, Y_{1}^{(1)}\right)^{\mathrm{BGK}}$ where $\left(b_{i}^{(1)}, Y_{1}^{(1)}\right)^{\mathrm{BGK}}$ is the corresponding coefficients and function for $\operatorname{Pr}=1$ [24]. Noting that $\gamma_{1}=\operatorname{Pr}$ for the ES model, we obtain a complete matching of $h_{D}, c_{1}$, and $c_{2}$ for the ES model with arbitrary $\operatorname{Pr}(\geqslant 2 / 3)$ and those for the BGK model by choosing $\operatorname{Pr} k^{\mathrm{ES}}=k^{\mathrm{BGK}}$, up to the order of $k$ where the slip coefficient $b_{1}^{(1)}$ appears for the first time in the asymptotic expressions. On the other hand, the jump coefficient $c_{3}^{(0)}$ is not self-similar for the ES model. Therefore, the coefficient $c_{3}$ cannot be matched by a similar conversion of $k$. There is no such exact correspondence between $\left(b_{i}^{(1)}, Y_{1}^{(1)}\right)$ for the hard-sphere model and that for the ES model. However, $b_{1}^{(1) \mathrm{ES}} \gamma_{1}^{\mathrm{HS}} / \operatorname{Pr}$ gives fairly close value to $b_{1}^{(1)}$ for the hard-sphere model when $\operatorname{Pr}=2 / 3$.

[39] Y. Sone and S. Takata, Discontinuity of the velocity distribution function in a rarefied gas around a convex body and the S layer at the bottom of the Knudsen layer, Transp. Theory Stat. Phys. 21, 501 (1992).

[40] S. Takata and S. Taguchi, Gradient divergence of fluid-dynamic quantities in rarefied gases on smooth boundaries, J. Stat. Phys. 168, 1319 (2017).

[41] H. Sugimoto and Y. Sone, Numerical analysis of steady flows of a gas evaporating from its cylindrical condensed phase on the basis of kinetic theory, Phys. Fluids A 4, 419 (1992).

[42] Y. Sone and H. Sugimoto, Steady evaporating flows from a spherical condensed phase into a vacuum, in Aerothermochemistry of Spacecraft and Associated Hypersonic Flows: Proceedings of the IUTAM Symposium Marseille 1992, edited by R. Brun and A. A. Chikhaoui (Jouve, Paris, 1994), p. 67.

[43] K. Aoki, K. Kanba, and S. Takata, Numerical analysis of a supersonic rarefied gas flow past a flat plate, Phys. Fluids 9, 1144 (1997).

[44] K. Aoki, S. Takata, H. Aikawa, and F. Golse, A rarefied gas flow caused by a discontinuous wall temperature, Phys. Fluids 13, 2645 (2001); 13, 3843(E) (2001). 